DOI 10.4171/JEMS/322

Christophe Mourougane

\title{
Families of hypersurfaces of large degree
}

\author{
Dedicated to Eckart Viehweg
}

Received October 19, 2009 and in revised form October 25, 2010 and January 25, 2012

Abstract. Grauert and Manin showed that a non-isotrivial family of compact complex hyperbolic curves has finitely many sections. We consider a general moving enough family of high enough degree hypersurfaces in a complex projective space. We show the existence of a strict closed subset of its total space that contains the images of all its sections.

\section{Introduction}

Grauert [Gra-65] and Manin [Man-63] solved the Mordell conjecture for curves over function fields. Lang generalised this statement in [Lang-86].

Conjecture (Lang's conjecture over function fields). Let $\pi: \mathcal{X} \rightarrow Y$ be a projective surjective morphism of complex algebraic manifolds, whose generic fibre is of general type. If $\pi$ is not birationally isotrivial, then there is a proper subscheme of $\mathcal{X}$ that contains the images of all sections of $\pi$.

Grauert's proof can be read as a construction of first order differential equations satisfied by all but a finite number of sections of the family $\pi$. First order differential equations are also enough to deal with families of manifolds with ample cotangent bundles ([Nog-81], [Mor-95]).

We implement this idea in higher dimensions with higher order differential equations, in the case where the positivity assumption is made only for the canonical bundle. We consider a family of hypersurfaces of $\mathbb{P}^{n+1}$ parametrised by a curve $C$ and given by a section of an ample line bundle $L=\lambda \otimes \mathcal{O}_{\mathbb{P}^{n+1}}(d)$ on $C \times \mathbb{P}^{n+1}$.

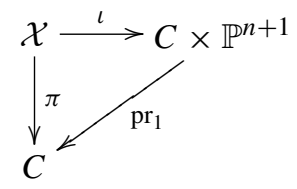

We will assume that the genus of $C$ and the relative dimension $n$ are at least 2. The degree of the hypersurfaces in the family is the integer $d$. We will say that the family is moving enough if $\operatorname{deg} \lambda$ is large. We prove

C. Mourougane: Institut de Recherche Mathématique de Rennes (IRMAR), Campus de Beaulieu, 35402 Rennes, France; e-mail: christophe.mourougane@univ-rennes1.fr 
Main Theorem. For a general moving enough family of high enough degree hypersurfaces of a complex projective space, there is a proper algebraic subset of the total space that contains the images of all its sections.

"General" refers to the family being chosen outside a proper algebraic subset of the parameter space. This assumption ensures in particular that the family is not birationally isotrivial.

By Noether's normalisation theorem and the primitive element theorem, every algebraic manifold $\mathcal{X}$ of dimension $n$ defined over the field $\mathbb{C}(Y)$ of rational functions on a connected manifold $Y$ is birational over $\mathbb{C}(Y)$ to a (usually singular) hypersurface in $\mathbb{P}_{\mathbb{C}(Y)}^{n+1}$.

We point out that Noguchi [Nog-85] gave a proof of Lang's conjecture when the smooth members of the family are hyperbolic under the assumption that the smooth part is hyperbolically embedded in the total space. Even though Kobayashi conjectured that a generic hypersurface of large degree of $\mathbb{P}^{n+1}$ is hyperbolic, our main result would not follow. Our proof does not rely on properties of families of hyperbolic manifolds, like normality. We benefit however from the recent works dealing with the Kobayashi conjecture, especially from [Dem-95].

The first part of our work (Section 2) describes general tools for dealing with higher order jets. The second part (Sections 3-5) is devoted to the proof of

Theorem 1. For every general moving enough family of high enough degree hypersurfaces of $\mathbb{P}^{n+1}$, there is a non-trivial differential equation of order $n+1$ satisfied by all its sections.

Then, adapting general techniques in universal families originating in the works of Clemens [Cle-86], Voisin [Voi-96] and Siu [Siu-04], we obtain the main theorem in the third part (Section 6).

\section{Jet spaces for sections}

Note that Lang's conjecture would follow from a positive answer to the case when the parameter space is a curve. We consider a proper morphism of complex manifolds $\pi$ : $\mathcal{X} \rightarrow C$, which we regard as a family of $n$-dimensional compact complex varieties parametrised by a connected compact complex smooth curve $C$. We intend to construct the jet spaces for the sections of $\pi$, allowing finite extensions of the base field $\mathbb{C}(C)$, that is, finite covers of the curve $C$.

\subsection{1-jets}

We follow the ideas of Grauert [Gra-65].

Consider a section $s: C_{\rho} \rightarrow \mathcal{X}$ of the pull-back family $\pi_{\rho}: \rho^{\star} \mathcal{X} \rightarrow C_{\rho}$, where $\rho: C_{\rho} \rightarrow C$ is a finite morphism of curves:

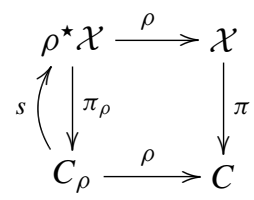


The map ${ }^{t} d s: s^{\star} \Omega_{\mathcal{X}} \rightarrow \Omega_{C_{\rho}}$ satisfies ${ }^{t} d s \circ s^{\star t} d \pi_{\rho}=\operatorname{Id}_{\Omega_{C_{\rho}}}$, so it is surjective and provides a rank one quotient of $s^{\star} \Omega_{\mathcal{X}}$. The corresponding curve $s_{1}: C_{\rho} \rightarrow \mathcal{X}_{\rho, 1}$ inside the bundle $\pi_{0, \rho, 1}: \mathcal{X}_{\rho, 1}:=\mathbb{P}\left(\rho^{\star} \Omega \mathcal{X}\right) \rightarrow \rho^{\star} \mathcal{X}$ of rank one quotients of $\rho^{\star} \Omega_{\mathcal{X}}$,

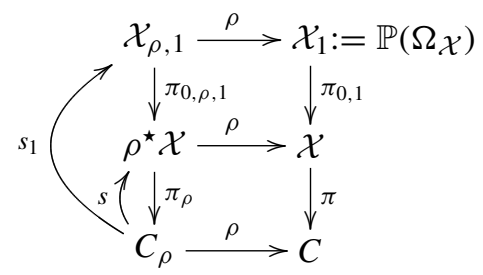

lifts $s$ (i.e. $\pi_{0, \rho, 1} \circ s_{1}=s$ ), is therefore a section of $\pi_{\rho, 1}: \mathcal{X}_{\rho, 1} \rightarrow C_{\rho}$. It avoids the divisor $\mathcal{D}_{1}:=\mathbb{P}\left(\Omega_{\mathcal{X} / C}\right)$ of vertical differentials, the divisor of the section of $\pi^{\star} T_{C} \otimes \mathcal{O}_{\Omega_{\mathcal{X}}}(1)$ given by ${ }^{t} d \pi: \pi^{\star} \Omega_{C} \rightarrow \Omega_{\mathcal{X}}$. We have to study the positivity properties of this line bundle, which translate into mobility properties of the forbidden divisor $\mathcal{D}_{1}$.

\subsection{2-jets}

The rest of the construction, which does not depend on the map $\pi$ but only on the total space $\mathcal{X}$, is due to Demailly-Semple [Dem-95]. We will omit the cover $\rho$. As in the preceding section, the curve $s_{1}: C \rightarrow \mathcal{X}_{1}$ lifts to a curve inside the bundle of rank one quotients of $\Omega_{\mathcal{X}_{1}}$. More precisely, the rank one quotient ${ }^{t} d s_{1}: s_{1}^{\star} \Omega_{\mathcal{X}_{1}} \rightarrow \Omega_{C}$ satisfies ${ }^{t} d s_{1} \circ s_{1}^{\star t} d \pi_{0,1}={ }^{t} d s$. The map ${ }^{t} d s_{1}$ at the point $\left[{ }^{t} d s\right]$ of $\mathcal{X}_{1}$ vanishes on the image under ${ }^{t} d \pi_{0,1}$ of the forms in the kernel of the tautological quotient ${ }^{t} d s$. In other words, ${ }^{t} d s_{1}$ is a rank one quotient of the quotient $\mathcal{F}_{1}$ of $\Omega_{\mathcal{X}_{1}}$ defined by the following diagram on $\mathcal{X}_{1}$ :

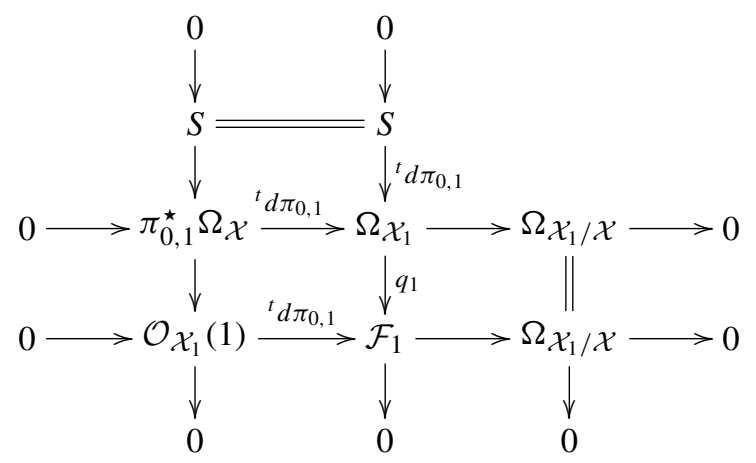

Define the 2-jet space to be $\pi_{1,2}: \mathcal{X}_{2}:=\mathbb{P}\left(\mathcal{F}_{1}\right) \rightarrow \mathcal{X}_{1}$. As in the formalism of Arrondo, Sols and Speiser [A-S-S-97], we need to keep track of the injective map $a_{2}=\mathbb{P}\left(q_{1}\right)$ : $\mathcal{X}_{2} \rightarrow \mathbb{P}\left(\Omega_{\mathcal{X}_{1}}\right)$ given by the quotient $q_{1}: \Omega_{\mathcal{X}_{1}} \rightarrow \mathcal{F}_{1}$ :<smiles>[Y10][Y10]1=[Y10][Y]1</smiles> 
We hence get a map $s_{2}: C \rightarrow \mathcal{X}_{2}$ defined by the quotient ${ }^{t} d s_{1}: s_{1}^{\star} \mathcal{F}_{1} \rightarrow \Omega_{C}$. Note that $\pi_{1,2}$ is the restriction to $\mathbb{P}\left(\mathcal{F}_{1}\right) \subset \mathbb{P}\left(\Omega_{\mathcal{X}_{1}}\right)$ of the map defined by the quotient $\pi_{1}^{\star} \Omega_{\mathcal{X}} \stackrel{{ }^{t} d \pi_{01}}{\longrightarrow}$ $\Omega \mathcal{X}_{1}$ so that the relation ${ }^{t} d s_{1} \circ s_{1}^{\star} d \pi_{0,1}={ }^{t} d s$ is rephrased by saying that the map $s_{2}$ is a lifting of $s_{1}$ (i.e. $\pi_{1,2} \circ s_{2}=s_{1}$ ):

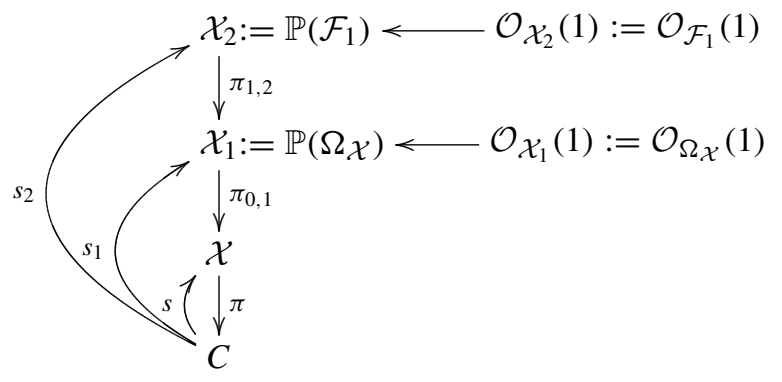

The map ${ }^{t} d \pi_{01}: \mathcal{O}_{\mathcal{X}_{1}}(1) \rightarrow \mathcal{F}_{1}$ gives rise to a section of $\pi_{1}^{\star} \mathcal{O}_{\mathcal{X}_{1}}(-1) \otimes \mathcal{O}_{\mathcal{X}_{2}}(1)$ whose divisor $\mathcal{D}_{2}:=\mathbb{P}\left(\Omega_{\mathcal{X}} / \mathcal{X}\right) \subset \mathcal{X}_{2}$ is not hit by the curve $s_{2}$ associated with the quotient ${ }^{t} d s_{1}$, as $\Omega_{C} \simeq s_{1}^{\star} \mathcal{O}_{\mathcal{X}_{1}}(1) \stackrel{s_{1}^{\star t} d \pi_{01}}{\longrightarrow} s_{1}^{\star} \mathcal{F}_{1} \stackrel{{ }^{t} d s_{1}}{\longrightarrow} \Omega_{C}$ vanishes nowhere.

\subsection{Higher order jets}

This scheme inductively leads to the construction of the $k$-jet space $\pi_{k-1, k}: \mathcal{X}_{k} \rightarrow \mathcal{X}_{k-1}$, together with a map $a_{k}: \mathcal{X}_{k} \rightarrow \mathbb{P}\left(\Omega_{\mathcal{X}_{k-1}}\right)$ that completes the commutative diagram

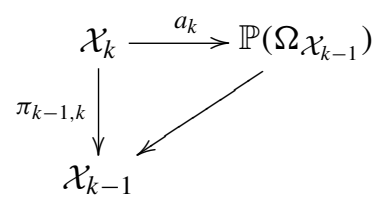

Note that $a_{k}^{\star} \mathcal{O}_{\Omega_{\mathcal{X}_{k-1}}}(1)=\mathcal{O}_{\mathcal{X}_{k}}(1)$. The bundle $\mathcal{F}_{k}$ on $\mathcal{X}_{k}$ is the quotient of $\Omega_{\mathcal{X}_{k}}$ defined by

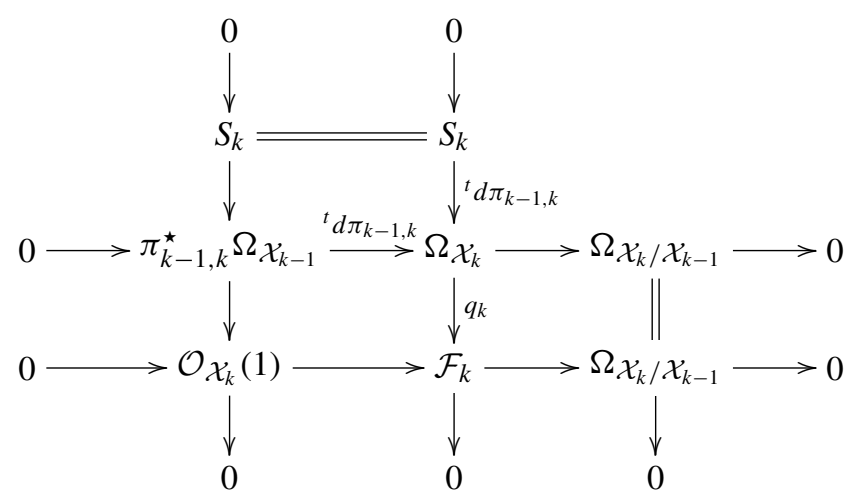


The $(k+1)$-jet space is $\pi_{k, k+1}: \mathcal{X}_{k+1}:=\mathbb{P}\left(\mathcal{F}_{k}\right) \rightarrow \mathcal{X}_{k}$ and the map $a_{k+1}: \mathcal{X}_{k+1} \rightarrow$ $\mathbb{P}\left(\Omega_{\mathcal{X}_{k}}\right)$ is the injective map associated with the quotient $q_{k}: \Omega_{\mathcal{X}_{k}} \rightarrow \mathcal{F}_{k}$. Note that the relative dimension of $\pi_{k+1, k}$ is equal to that of $\pi_{k-1, k}$, which is $n$. Therefore

$$
\operatorname{dim} \mathcal{X}_{k}=(k+1) n+1 \text {. }
$$

Now, given a section $s: C \rightarrow \mathcal{X}$ of the family $\pi: \mathcal{X} \rightarrow C$, assuming that we have constructed the lifts $s_{i}: C \rightarrow \mathcal{X}_{i}$ up to level $k$, we get the $(k+1)$-jet $s_{k+1}$ : $C \rightarrow \mathcal{X}_{k+1}$ by considering the surjective map ${ }^{t} d s_{k}: s_{k}^{\star} \mathcal{F}_{k} \rightarrow \Omega_{C}$ built from the relation ${ }^{t} d s_{k} \circ s_{k}^{\star t} d \pi_{k-1, k}={ }^{t} d s_{k-1}$. Recall that the tautological quotient bundle $\mathcal{O}_{\mathcal{X}_{k+1}}$ (1) pulls back to $C$ via $s_{k+1}$ into the quotient $\Omega_{C}$ under consideration:

$$
s_{k+1}^{\star} \mathcal{O}_{\mathcal{X}_{k+1}}(1)=\Omega_{C} .
$$

The map ${ }^{t} d \pi_{k-1, k}: \mathcal{O}_{\mathcal{X}_{k}}(1) \rightarrow \mathcal{F}_{k}$ gives rise to a divisor $\mathcal{D}_{k+1}=\mathbb{P}\left(\Omega_{\mathcal{X}_{k} / \mathcal{X}_{k-1}}\right)$ in $\mathcal{X}_{k+1}=\mathbb{P}\left(\mathcal{F}_{k}\right)$ in the linear system $\left|\pi_{k+1, k}^{\star} \mathcal{O}_{\mathcal{X}_{k}}(-1) \otimes \mathcal{O}_{\mathcal{X}_{k+1}}(1)\right|$ that the curve $s_{k+1}$ avoids.

\subsection{Description in coordinates}

Choose a local coordinate $t$ on $C$ and an adapted system of local coordinates $\left(t, z_{1}, \ldots, z_{n}\right)$ on $\mathcal{X}$ at a regular point of the map $\pi$ such that the map $\pi$ is given by $\left(t, z_{1}, \ldots, z_{n}\right) \mapsto t$. The set of vectors $\partial / \partial t, \partial / \partial z_{1}, \ldots, \partial / \partial z_{n}$ provides a local frame for $T_{\mathcal{X}}$. This defines relative homogeneous coordinates $\left[T_{1}: A_{1}: \cdots: A_{n}\right]$ on $\mathcal{X}_{1}$.

The differential of a section $s$ of $\pi$ locally written as $t \mapsto\left(t, z_{1}(t), z_{2}(t), \ldots, z_{n}(t)\right)$ is

$$
d s: \frac{\partial}{\partial t} \mapsto \frac{\partial}{\partial t}+z_{1}^{\prime}(t) \frac{\partial}{\partial z_{1}}+\cdots+z_{n}^{\prime}(t) \frac{\partial}{\partial z_{n}} .
$$

The 1-jet of the curve $s$ is therefore locally written as $s_{1}: C \rightarrow \mathcal{X}_{1}=P\left(T_{\mathcal{X}}\right)$,

$$
s_{1}: t \mapsto\left(t, z_{1}(t), \ldots, z_{n}(t),\left[1: z_{1}^{\prime}(t): \cdots: z_{n}^{\prime}(t)\right]\right)
$$

It does not meet the divisor $\mathcal{D}_{1}:=P\left(T_{\mathcal{X} / C}\right)$ locally given by $T_{1}=0$.

Outside this divisor, we get relative affine coordinates $a_{1}:=A_{1} / T_{1}, \ldots, a_{n}:=A_{n} / T_{1}$. Note that for the section $s_{1}$ we infer that $a_{j}(t)=z_{j}^{\prime}(t)$. The set of vectors

$$
\frac{\partial}{\partial t}, \frac{\partial}{\partial z_{1}}, \ldots, \frac{\partial}{\partial z_{n}}, \frac{\partial}{\partial a_{1}}, \ldots, \frac{\partial}{\partial a_{n}}
$$

provides a local frame for $T_{\mathcal{X}_{1}}$. The bundle $\mathcal{F}_{1}^{\star}$ is defined to be

$$
\mathcal{F}_{1}^{\star}:=\left\{(t, z,[A], v) \in T_{\mathcal{X}_{1}} \mid d \pi_{0,1}(v) \in[A] \subset T_{\mathcal{X}}\right\} .
$$

It has a local frame built with $\partial / \partial t+a_{1} \partial / \partial z_{1}+\cdots+a_{n} \partial / \partial z_{n} \in \mathcal{O}_{\mathcal{X}_{1}}(-1)$ and $\partial / \partial a_{i} \in$ $T_{\mathcal{X}_{1} / \mathcal{X}}, 1 \leq i \leq n$. This defines relative homogeneous coordinates $\left[T_{2}: B_{1}: \cdots: B_{n}\right]$ on $\mathcal{X}_{2}$. 
The differential of the section $s_{1}: C \rightarrow \mathcal{X}_{1}-\mathcal{D}_{1}$ of $\pi_{1}$ is

$$
\begin{aligned}
d s_{1}: \frac{\partial}{\partial t} \mapsto \frac{\partial}{\partial t}+ & z_{1}^{\prime}(t) \frac{\partial}{\partial z_{1}}+\cdots+z_{n}^{\prime}(t) \frac{\partial}{\partial z_{n}}+z_{1}^{\prime \prime}(t) \frac{\partial}{\partial a_{1}}+\cdots+z_{n}^{\prime \prime}(t) \frac{\partial}{\partial a_{n}} \\
& =\left(\frac{\partial}{\partial t}+a_{1}(t) \frac{\partial}{\partial z_{1}}+\cdots+a_{n}(t) \frac{\partial}{\partial z_{n}}\right)+z_{1}^{\prime \prime}(t) \frac{\partial}{\partial a_{1}}+\cdots+z_{n}^{\prime \prime}(t) \frac{\partial}{\partial a_{n}} .
\end{aligned}
$$

The 2-jet $s_{2}: C \rightarrow \mathcal{X}_{2}$ is locally written as

$$
s_{2}: t \mapsto\left(t, z_{1}(t), \ldots, z_{n}(t),\left[1: z_{1}^{\prime}(t): \cdots: z_{n}^{\prime}(t)\right],\left[1: z_{1}^{\prime \prime}(t): \cdots: z_{n}^{\prime \prime}(t)\right]\right) .
$$

It does not meet the divisor $\mathcal{D}_{2}:=P\left(T_{\mathcal{X}_{2} / \mathcal{X}_{1}}\right)$ locally given by $T_{2}=0$.

Coordinates in higher order jet spaces are defined similarly, over the regular points of the family $\pi$.

\section{Vanishing criterion and algebraic Morse inequalities}

This section is devoted to the tools needed to prove Theorem 1 on the existence of differential equations satisfied by all sections of the given family $\mathcal{X} \rightarrow C$ or its pull-back $\rho^{\star} \mathcal{X} \rightarrow C_{\rho}$ under a finite morphism $\rho: C_{\rho} \rightarrow C$.

\subsection{Vanishing criterion}

Consider the line bundles on the $k$-jet space $\mathcal{X}_{k}$ defined by

$$
\mathcal{O}_{\mathcal{X}_{k}}(\underline{m}):=\pi_{1, k}^{\star} \mathcal{O}_{\mathcal{X}_{1}}\left(m_{1}\right) \otimes \pi_{2, k}^{\star} \mathcal{O}_{\mathcal{X}_{2}}\left(m_{2}\right) \otimes \cdots \otimes \mathcal{O}_{\mathcal{X}_{k}}\left(m_{k}\right)
$$

and

$$
\mathcal{O}_{\mathcal{X}_{k}}(\underline{M \mathcal{D}}):=\pi_{1, k}^{\star} \mathcal{O}_{\mathcal{X}_{1}}\left(M_{1} \mathcal{D}_{1}\right) \otimes \pi_{2, k}^{\star} \mathcal{O}_{\mathcal{X}_{2}}\left(M_{2} \mathcal{D}_{2}\right) \otimes \cdots \otimes \mathcal{O}_{\mathcal{X}_{k}}\left(M_{k} \mathcal{D}_{k}\right)
$$

Define

$$
\begin{aligned}
\chi_{\rho} & :=\int_{C_{\rho}} \operatorname{seg}_{1}\left(\Omega_{C_{\rho}}\right)=-\int_{C_{\rho}} c_{1}\left(T_{C_{\rho}}\right)=-2 \int_{C_{\rho}} \operatorname{Todd}\left(T_{C_{\rho}}\right)=-2 \chi\left(C_{\rho}\right) \\
& =2 g\left(C_{\rho}\right)-2 \geq(\operatorname{deg} \rho)(2 g(C)-2) \geq 0 .
\end{aligned}
$$

Take a line bundle $\mu$ on the base curve $C$. Consider a section $\sigma$ of the line bundle

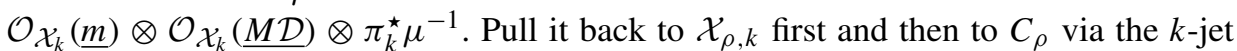
$s_{k}: C_{\rho} \rightarrow \mathcal{X}_{\rho, k}$ of a section $s$ of the pulled-back family $\rho^{\star} \mathcal{X} \rightarrow C_{\rho}$. This gives a section $s_{k}^{\star} \sigma$ of the line bundle $\Omega_{C_{\rho}}^{\otimes|\underline{m}|} \otimes \rho^{\star} \mu^{-1}$. If the latter bundle has an ample dual bundle (i.e. if $\left.\operatorname{deg} \rho \operatorname{deg} \mu>|\underline{m}| \chi_{\rho}\right)$, then the section $s_{k}^{\star} \sigma$ has to vanish. This gives 
Lemma 3.1 (The vanishing criterion). If a line bundle $\mu$ on the base curve $C$ has

$$
\operatorname{deg} \mu>|\underline{m}| \frac{\chi \rho}{\operatorname{deg} \rho}
$$

then for every section $\sigma$ of (a multiple of ) the line bundle $\mathcal{O}_{\mathcal{X}_{k}}(\underline{m}) \otimes \mathcal{O}_{\mathcal{X}_{k}}(\underline{M \mathcal{D}}) \otimes \pi_{k}^{\star} \mu^{-1}$ on $\mathcal{X}_{k}$ and every section s of the pulled-back family $\rho^{\star} \mathcal{X} \rightarrow C_{\rho}$, the $k$-jet $s_{k}$ of $s$ lies in the zero locus of $\rho^{\star} \sigma$,

$$
s_{k}\left(C_{\rho}\right) \subset \operatorname{Zero}\left(\rho^{\star} \sigma\right) \subset \mathcal{X}_{\rho, k} .
$$

Note that we have considered only those bundles having zero components along the Picard group of $\mathcal{X} / C$. For example, in the case of a family of hypersurfaces of $\mathbb{P}^{n+1}$, bounding the intersection number $s(C) \cdot \mathcal{O}_{\mathbb{P}^{n+1}}(1)$, called the height of the section $s$, is a main step in proving Lang's conjecture. We could alternatively allow a negative part along the Picard group of $\mathcal{X} / C$. This will give the height estimates in Section 5.

\subsection{Algebraic Morse inequalities}

We therefore have to try to produce sections of bundles with negative components along the Picard group of $\mathcal{X} / C$. We will use the algebraic form of holomorphic Morse inequalities [Dem-85], [Tra-95] to achieve this.

Proposition 3.2 (Algebraic Morse inequalities). Suppose a line bundle L on a projective manifold of dimension $D$ can be written as the difference of two nef line bundles, $L=A-B$, where the intersection number $A^{D}-D A^{D-1} \cdot B$ is positive. Then $L$ is big.

There are three elements to settle to get the proof of Theorem 1: first, the construction of nef line bundles $A$ and $B$ on a jet space $\mathcal{X}_{k}$ with $L=A-B$ having negative component on $\operatorname{Pic}\left(\mathcal{X}_{k} / C\right)$ and being of weight $\underline{m}$; secondly, the inequality $\operatorname{deg} \mu>\chi_{\rho}|\underline{m}| / \operatorname{deg} \rho$ for the negative part $\mu^{-1}$ of $L$ coming from the base curve $C$ needed to apply the vanishing criterion; and finally the positivity of the intersection number $A^{\operatorname{dim} \mathcal{X}_{k}}-\operatorname{dim} \mathcal{X}_{k} A^{\operatorname{dim} \mathcal{X}_{k}-1} \cdot B$.

\section{The nef cones}

From now on we will restrict to the situation of a family of hypersurfaces in $\mathbb{P}^{n+1}$ given by a section $F_{0}$ of an ample line bundle $L_{0}$ on $C \times \mathbb{P}^{n+1}$. We will assume that the genus of $C$ and the relative dimension $n$ are at least 2 .

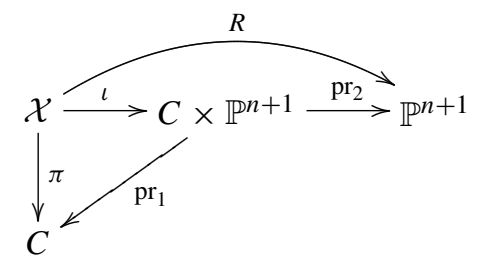

This gives a further sequence on $\mathcal{X}$

$$
0 \rightarrow L_{0 \mid \mathcal{X}}^{\star} \stackrel{{ }^{t} d F_{0}}{\longrightarrow} \Omega_{C} \boxplus \Omega_{\mathbb{P}^{n+1} \mid \mathcal{X}} \stackrel{{ }^{t} d \iota}{\longrightarrow} \Omega_{\mathcal{X}}=\mathcal{F}_{0} \rightarrow 0 .
$$


From the Leray-Hirsch theorem, we know that $\operatorname{Pic}\left(C \times \mathbb{P}^{n+1}\right)=\operatorname{pr}_{1}^{\star} \operatorname{Pic} C \oplus \operatorname{pr}_{2}^{\star} \operatorname{Pic}^{n+1}$. In particular, we will write $L_{0}$ as $\lambda_{0} \otimes \mathcal{O}_{\mathbb{P}^{n+1}}\left(d_{0}\right)=\operatorname{pr}_{1}^{\star} \lambda_{0} \otimes \operatorname{pr}_{2}^{\star} \mathcal{O}_{\mathbb{P}^{n+1}}\left(d_{0}\right)$. Note that $\mathcal{O}_{\mathbb{P}^{n+1}}\left(d_{0}\right)=\left(L_{0}\right)_{\mid \mathrm{pr}_{1}^{-1} b}$ is ample $\left(d_{0}>0\right)$ and $\left(\mathrm{pr}_{1}\right)_{\star} L_{0}=\lambda_{0} \otimes S^{d_{0}} \mathbb{C}^{n+2}$ is effective $\left(\operatorname{deg} \lambda_{0} \geq 0\right)$.

\subsection{The nef cone of $\mathcal{X}$}

As the line bundle $L_{0}$ is assumed to be ample and $\mathcal{X}$ is of dimension at least 3 , the Lefschetz hyperplane theorem reads

$$
\operatorname{Pic} \mathcal{X}=\iota^{\star} \operatorname{Pic}\left(C \times \mathbb{P}^{n+1}\right)=\pi^{\star} \operatorname{Pic} C \oplus R^{\star} \operatorname{Pic} \mathbb{P}^{n+1} .
$$

For a line bundle $\lambda$ on $C$ and an integer $d$, we will denote by $\mathcal{O}_{\mathcal{X}}(\lambda, d)=\pi^{\star} \lambda \otimes$ $R^{\star} \mathcal{O}_{\mathbb{P}^{n+1}}(d)$ the restriction to $\mathcal{X}$ of the line bundle $\lambda \otimes \mathcal{O}_{\mathbb{P} n+1}(d)$.

The line bundle $\pi^{\star} \mathcal{O}_{B}(1)$, nef but not ample, has its Chern class lying on a vertex of the nef cone of $\mathcal{X}$. If the morphism $R: \mathcal{X} \rightarrow \mathbb{P}^{n+1}$ is not finite (e.g. the section defining $\mathcal{X}$ does not involve all the homogeneous coordinates on $\mathbb{P}^{n+1}$ ) then the line bundle $R^{\star} \mathcal{O}_{\mathbb{P}^{n+1}}(1)$ gives the second vertex. This is not the general case.

The top intersection number of the first Chern class $c_{1}\left(\mathcal{O}_{\mathcal{X}}(\lambda, d)\right) \in \mathrm{NS}(\mathcal{X})$ is given by

$$
\begin{aligned}
c_{1}\left(\mathcal{O}_{\mathcal{X}}\right. & (\lambda, d))^{n+1} \\
& =\iota^{\star}\left[\operatorname{pr}_{1}^{\star} c_{1}(\lambda)+\operatorname{pr}_{2}^{\star} c_{1}\left(\mathcal{O}_{\mathbb{P}^{n+1}}(d)\right)\right]^{n+1} \\
& =\left[c_{1}\left(\lambda_{0}\right)+c_{1}\left(\mathcal{O}_{\mathbb{P}^{n+1}}\left(d_{0}\right)\right)\right] \cdot\left[c_{1}\left(\mathcal{O}_{\mathbb{P}^{n+1}}(d)\right)^{n+1}+(n+1) c_{1}(\lambda) \operatorname{pr}_{2}^{\star} c_{1}\left(\mathcal{O}_{\mathbb{P}^{n+1}}(d)\right)^{n}\right] \\
& =d^{n}\left[d \operatorname{deg} \lambda_{0}+(n+1) d_{0} \operatorname{deg} \lambda\right] .
\end{aligned}
$$

It has to be non-negative on the nef cone. We hence get, in $\mathrm{NS}_{\mathbb{R}}(\mathcal{X}) \equiv \mathbb{R}^{2}$,

$$
\begin{aligned}
\{(l, d) \mid d \geq 0, l \geq 0\} & =\iota^{\star} \operatorname{Nef}\left(B \times \mathbb{P}^{n+1}\right) \\
& \subset \operatorname{Nef}(\mathcal{X}) \subset\left\{(l, d) \mid d \geq 0, l \geq-\frac{\operatorname{deg} \lambda_{0}}{n+1} \frac{d}{d_{0}}\right\} .
\end{aligned}
$$

\subsection{The pseudo-effective cone of $\mathcal{X}$}

We now compute the pseudo-effective cone, $\operatorname{Psef}(\mathcal{X}) \supset \operatorname{Nef}(\mathcal{X})$. Take $\operatorname{deg} \lambda<0$ and $d>0$. The push-forward by $\mathrm{pr}_{1}$ of the sequence defining the structure sheaf of $\mathcal{X}$ tensorised by $\lambda \otimes \mathcal{O}_{\mathbb{P}^{n+1}}(d)$ reads

$$
0 \rightarrow\left(\mathrm{pr}_{1}\right)_{\star}\left(\lambda \otimes \lambda_{0}^{\star} \otimes \mathcal{O}_{\mathbb{P}^{n+1}}\left(d-d_{0}\right)\right) \rightarrow\left(\mathrm{pr}_{1}\right)_{\star}\left(\lambda \otimes \mathcal{O}_{\mathbb{P}^{n+1}}(d)\right) \rightarrow \pi_{\star} \mathcal{O}_{\mathcal{X}}(\lambda, d) \rightarrow 0,
$$

that is,

$$
0 \rightarrow \lambda \otimes \lambda_{0}^{\star} \otimes S^{d-d_{0}} \mathbb{C}^{n+2} \rightarrow \lambda \otimes S^{d} \mathbb{C}^{n+2} \rightarrow \pi_{\star} \mathcal{O}_{\mathcal{X}}(\lambda, d) \rightarrow 0
$$


As $\operatorname{deg} \lambda<0$, the associated long exact sequence gives

$$
\begin{aligned}
& 0 \rightarrow H^{0}\left(\mathcal{X}, \mathcal{O}_{\mathcal{X}}(\lambda, d)\right) \\
& \rightarrow H^{1}\left(C, \lambda \otimes \lambda_{0}^{\star}\right) \otimes S^{d-d_{0}} \mathbb{C}^{n+2} \rightarrow H^{1}(C, \lambda) \otimes S^{d} \mathbb{C}^{n+2} \rightarrow H^{1}\left(C, \pi_{\star} \mathcal{O}_{\mathcal{X}}(\lambda, d)\right) \rightarrow 0 .
\end{aligned}
$$

Note that if $\ell$ is large, the higher direct image $\mathcal{R}^{1} \pi_{\star} \mathcal{O}_{\mathcal{X}}\left(\lambda^{\otimes \ell}, \ell d\right)$ vanishes, so that $H^{1}\left(C, \pi_{\star} \mathcal{O}_{\mathcal{X}}\left(\lambda^{\otimes \ell}, \ell d\right)\right)$ and $H^{1}\left(\mathcal{X}, \mathcal{O}_{\mathcal{X}}\left(\lambda^{\otimes \ell}, \ell d\right)\right)$ become isomorphic. We infer

$$
\begin{aligned}
h^{0}\left(\mathcal{X}, \mathcal{O}_{\mathcal{X}}(\lambda, d)\right) \geq & h^{1}\left(C, \lambda \otimes \lambda_{0}^{\star}\right) \otimes S^{d-d_{0}} \mathbb{C}^{n+2}-h^{1}(C, \lambda) \otimes S^{d} \mathbb{C}^{n+2} \\
\geq & -\chi\left(C, \lambda \otimes \lambda_{0}^{\star}\right) \otimes S^{d-d_{0}} \mathbb{C}^{n+2}+\chi(C, \lambda) \otimes S^{d} \mathbb{C}^{n+2} \\
\geq & {[\operatorname{deg} \lambda+1-g(C)]\left(\begin{array}{c}
d+n+1 \\
n+1
\end{array}\right) } \\
& -\left[\operatorname{deg} \lambda-\operatorname{deg} \lambda_{0}+1-g(C)\right]\left(\begin{array}{c}
d-d_{0}+n+1 \\
n+1
\end{array}\right) .
\end{aligned}
$$

We find that if

$$
\operatorname{deg} \lambda>-\frac{\operatorname{deg} \lambda_{0}}{n+1} \frac{d}{d_{0}}
$$

then for large $\ell, H^{0}\left(\mathcal{X}, \mathcal{O}_{\mathcal{X}}\left(\lambda^{\otimes \ell}, \ell d\right)\right) \neq 0$. Hence

$$
\{d \geq 0, l \geq 0\} \subset \operatorname{Nef}(\mathcal{X}) \subset\left\{d \geq 0, l \geq-\frac{\operatorname{deg} \lambda_{0}}{n+1} \frac{d}{d_{0}}\right\} \subset \operatorname{Psef}(\mathcal{X}) .
$$

\subsection{The cones in the very general case}

The ideas described here are due to Claire Voisin. The key result is the following

Lemma 4.1. Let $\mathcal{Y} \subset T \times P \rightarrow T$ be a family of complex algebraic ample hypersurfaces of dimension at least 3 of a projective manifold $P$. Assume that the fibre $Y_{0}$ over 0 in $T$ is irreducible and that the nef cone and the pseudo-effective cone of its normalisation coincide. Then the nef cone and the pseudo-effective cone of a very general fibre of $\mathcal{Y} \rightarrow T$ also coincide.

"Very general" refers to the family being chosen outside a countable union of proper algebraic subsets of the parameter space.

Proof. The Picard group of any general member $Y_{t}$ is induced by that of $P$ by the Lefschetz theorem. Take a numerical class $c$ in $\mathrm{NS}(P)$ and a line bundle $\mathcal{L}$ on $P$ in the class $c$. Using semicontinuity theorems for the universal bundle over $\operatorname{Pic}^{0}(\mathcal{Y} / T)$ twisted by $\mathcal{L}$ and the properness and flatness of the relative Picard scheme $\operatorname{Pic}^{0}(\mathcal{Y} / T) \rightarrow T$ over the smooth locus of $\mathcal{Y} \rightarrow T$, we see that the locus $Z_{c}$ of $T$ where the line bundle $\mathcal{L}_{\mid Y_{t}}$ is algebraically equivalent to an effective line bundle is Zariski closed. Define $Z$ to be the non-smooth locus of $\mathcal{Y} \rightarrow T$ together with the countable union of all those $Z_{c}$ that are strict in $T$. Removing the countable union $Z^{\prime}$ of images in $T$ of components of the Hilbert scheme of vertical curves in $\mathcal{Y}$ that do not dominate $T$, we can ensure that every curve $C$ in $Y_{t}$ 
for $t \in T-Z^{\prime}$ deforms locally around $t$, and by properness of components of the Hilbert schemes, specialises to a curve $C_{0}$ at 0 .

Take a $\tau \in T-Z-Z^{\prime}$. Take a line bundle $\mathcal{L} \in \operatorname{Pic}(P)$ whose restriction to $Y_{\tau}$ is effective and a curve $C$ in $Y_{\tau}$. We have to check that the degree $\operatorname{deg} \mathcal{L}_{\mid C}$ is non-negative. The line bundle $\mathcal{L}_{\mid Y_{t}}$ is algebraically equivalent to an effective line bundle on the whole of $T-Z-Z^{\prime}$ and therefore $\mathcal{L}_{\mid Y_{0}}$ pulls back to a nef line bundle on the normalisation of $Y_{0}$, by hypothesis. Here we use the irreducibility of $Y_{0}$ to make sure that the section obtained does not identically vanish on some irreducible component of $Y_{0}$. As $\operatorname{deg} v^{\star} \mathcal{L}_{\mid v^{-1} C_{0}} \geq 0$, we infer, using intersection theory for line bundles on the singular fibre $Y_{0}$ and especially the projection formula, that the integer $\operatorname{deg} \mathcal{L}_{\mid C}$ is non-negative.

In our setting, under an assumption on the shape of the defining line bundle $L_{0}$, this leads to

Proposition 4.2. Take a line bundle $L_{0}=\lambda_{0}^{n+1} \otimes \mathcal{O}_{\mathbb{P}^{n+1}}\left(d_{0}\right)$ on $C \times \mathbb{P}^{n+1}$, where $\lambda_{0}$ is a line bundle on $C$ having a pencil of sections and $d_{0}$ is any positive integer. If $\mathcal{X}$ is general in the linear system $\left|L_{0}\right|$, then

$$
\operatorname{Nef}(\mathcal{X})=\operatorname{Psef}(\mathcal{X})=\left\{(l, d) \mid d \geq 0, l \geq-\operatorname{deg} \lambda_{0} \frac{d}{d_{0}}\right\}
$$

Proof. Take a rational function $f: C \rightarrow \mathbb{P}^{1}$ given by a pencil of sections of $\lambda_{0}$ and a generic hypersurface $X$ of $\mathbb{P}^{n+1}$ defined by a polynomial $F$ of degree $d_{0}$. Construct the finite map from the Segre embedding and a generic projection

$$
\phi: C \times X \rightarrow \mathbb{P}^{1} \times \mathbb{P}^{n+1} \rightarrow \mathbb{P}^{2 n+1} \rightarrow \mathbb{P}^{n+1},
$$

and consider the map $\Phi=\left(\operatorname{Id}_{C}, \phi\right): C \times X \rightarrow C \times \mathbb{P}^{n+1}$. Denote its image by $\mathcal{X}_{0}$. The $\operatorname{map} \mathbb{P}^{1} \times \mathbb{P}^{n+1} \rightarrow \mathbb{P}^{2 n+1} \rightarrow \mathbb{P}^{n+2}$ is explicitly given in coordinates by

$$
\begin{array}{r}
\left(\left[X_{0}: X_{1}\right],\left[Y_{0}: Y_{1}: \cdots: Y_{n+1}\right]\right) \mapsto\left[2 X_{1} Y_{0}: X_{0} Y_{0}-X_{1} Y_{1}: X_{0} Y_{1}-X_{1} Y_{2}: \cdots\right. \\
\left.\cdots: X_{0} Y_{n}-X_{1} Y_{n+1}: 2 X_{0} Y_{n+1}\right] .
\end{array}
$$

If $F(1,0,0,0) \neq 0$ and $F$ is generic, we can project to get a finite map $\mathbb{P}^{1} \times X \rightarrow \mathbb{P}^{n+1}$,

$$
\begin{aligned}
&\left(\left[X_{0}: X_{1}\right],\left[Y_{0}: Y_{1}: \cdots: Y_{n+1}\right]\right) \mapsto\left[X_{0} Y_{0}-\right. X_{1} Y_{1}: X_{0} Y_{1}-X_{1} Y_{2}: \cdots \\
&\left.\cdots: X_{0} Y_{n}-X_{1} Y_{n+1}: 2 X_{0} Y_{n+1}\right] .
\end{aligned}
$$

The equation of the image $\mathcal{X}_{0}$ of $\Phi$,

$$
\begin{aligned}
& F\left(X_{0}^{n+1} U_{0}+X_{0}^{n} X_{1} U_{1}+X_{0}^{n-1} X_{1}^{2} U_{2}+\cdots+X_{1}^{n+1} \frac{U_{n+1}}{2}: \cdots\right. \\
& \left.\cdots: X_{0}^{n+1} U_{n-1}+X_{0}^{n} X_{1} U_{n}+X_{0}^{n-1} X_{1}^{2} \frac{U_{n+1}}{2}: X_{0}^{n+1} U_{n}+X_{0}^{n} X_{1} \frac{U_{n+1}}{2}: X_{0}^{n+1} \frac{U_{n+1}}{2}\right)=0,
\end{aligned}
$$

is in the linear system $\left|\lambda_{0}^{(n+1) d_{0}} \otimes \mathcal{O}_{\mathbb{P}^{n+1}}\left(d_{0}\right)\right|$. Note that $n+1$ is the degree of the image of $\mathbb{P}^{1} \times \mathbb{P}^{n}$, considered as a divisor in $\mathbb{P}^{1} \times \mathbb{P}^{n+1}$, under the Segre map to $\mathbb{P}^{2 n+1}$. 
The normalisation of $\mathcal{X}_{0}$ is $C \times X$ whose nef cone is equal to its effective cone. By Lemma 4.1, we infer that the same holds true for very general deformations of the image $\mathcal{X}_{0}$.

We can now apply this to get more linear systems than just those of type $\mid \lambda_{0}^{(n+1) d_{0}} \otimes$ $\mathcal{O}_{\mathbb{P}^{n+1}}\left(d_{0}\right) \mid$. Take $\mathcal{X}$ to be a general hypersurface in the linear system $\left|\lambda_{0}^{(n+1)} \otimes \mathcal{O}_{\mathbb{P}^{n+1}}(1)\right|$ whose nef cone and the pseudo-effective cone coincide. Consider the Frobenius like finite morphism $\psi: \mathbb{P}^{n+1} \rightarrow \mathbb{P}^{n+1}$ obtained by raising homogeneous coordinates to the power $\delta_{0}$, and the hypersurface $\mathcal{X}^{\prime}:=\left(\operatorname{Id}_{\mid B}, \psi\right)^{-1}(\mathcal{X})$. It is a smooth ample hypersurface of $C \times \mathbb{P}^{n+1}$ in the linear system $\left|\lambda_{0}^{(n+1)} \otimes \mathcal{O}_{\mathbb{P}^{n+1}}\left(\delta_{0}\right)\right|$. By the Lefschetz theorem, its $\mathbb{Q}$ Néron-Severi group coincides with that of $C \times \mathbb{P}^{n+1}$. Take a curve $C^{\prime}$ and an effective divisor $D^{\prime}$ in $\mathcal{X}^{\prime}$. Its multiple $\delta_{0} D^{\prime}$ pulls back from an effective divisor $D$ in $\mathcal{X}$, which is nef by hypothesis, and

$$
\delta_{0} D^{\prime} \cdot C^{\prime}=\psi^{-1}(D) \cdot C^{\prime}=\delta_{0}^{n+1} D \cdot \psi\left(C^{\prime}\right) \geq 0 .
$$

The hypersurface $\mathcal{X}^{\prime}$ may not be general, but applying the lemma again, we infer that the nef cone and the pseudo-effective cone of a very general hypersurface in the linear system $\left|\lambda_{0}^{(n+1)} \otimes \mathcal{O}_{\mathbb{P}^{n+1}}\left(\delta_{0}\right)\right|$ coincide.

\subsection{A nef line bundle on $\mathcal{X}_{1}$}

By the Leray-Hirsch theorem, the Picard group of $\mathcal{X}_{1}=\mathbb{P}\left(\mathcal{F}_{0}\right)$ is

$$
\operatorname{Pic} \mathcal{X}_{1}=\operatorname{Pic} \mathcal{X} \oplus \mathbb{Z} \mathcal{O}_{\mathcal{X}_{1}}(1)=\operatorname{Pic} C \oplus \operatorname{Pic} \mathbb{P}^{n+1} \oplus \mathbb{Z} \mathcal{O}_{\mathcal{X}_{1}}(1) \text {. }
$$

Accordingly, we will use the notation $\mathcal{O}_{\mathcal{X}_{1}}\left(\lambda, d ; m_{1}\right)$. The bundle $\Omega_{\mathbb{P} n+1}=\Lambda^{n} T_{\mathbb{P}^{n+1}} \otimes$ $K_{\mathbb{P}^{n+1}}$ is a quotient of $\left(\Lambda^{n} \mathcal{O}_{\mathbb{P}^{n+1}}(1)^{\oplus n+1}\right) \otimes K_{\mathbb{P}^{n+1}}=\left(\Lambda^{n} \mathcal{O}_{\mathbb{P} n+1}^{\oplus n+1}\right) \otimes \mathcal{O}_{\mathbb{P}^{n+1}}(-2)$. Hence, as $\Omega_{C}$ is globally generated, the quotient (see 4.1) $\mathcal{F}_{0} \otimes \mathcal{O}_{\mathbb{P} n+1}(2)$ and therefore the bundle $\mathcal{L}_{1}:=\mathcal{O}_{\mathcal{X}_{1}}(0,2 ; 1)$ are also globally generated.

\subsection{A nef line bundle on $\mathcal{X}_{k+1}$}

Generally, the bundle $\Omega \mathcal{X}_{k} / \mathcal{X}_{k-1}=\Lambda^{n-1} T_{\mathcal{X}_{k} / \mathcal{X}_{k-1}} \otimes K_{\mathcal{X}_{k}} / \mathcal{X}_{k-1}$ is a quotient of

$$
\begin{array}{r}
\Lambda^{n-1}\left(\pi_{k-1, k}^{\star} \mathcal{F}_{k-1}^{\star} \otimes \mathcal{O}_{\mathcal{X}_{k}}(1)\right) \otimes \mathcal{O}_{\mathcal{X}_{k}}(-n-1) \otimes \pi_{k-1, k}^{\star} \operatorname{det} \mathcal{F}_{k-1} \\
=\pi_{k-1, k}^{\star} \mathcal{F}_{k-1} \otimes \mathcal{O}_{\mathcal{X}_{k}}(-2)
\end{array}
$$

Assuming that $\mathcal{O}_{\mathcal{X}_{k-1}}\left(m_{k-1}\right)$ and $\mathcal{O}_{\mathcal{X}_{k}}\left(m_{k-1}, 1\right)$ are nef, we infer from the defining sequence of $\mathcal{F}_{k}$,

$$
\begin{aligned}
0 \rightarrow \mathcal{O}_{\mathcal{X}_{k}}\left(\underline{3 m_{k-1}}, 3\right) \rightarrow \mathcal{F}_{k} \otimes \mathcal{O}_{\mathcal{X}_{k}}(2) \otimes \pi_{k-1, k}^{\star} \mathcal{O}_{\mathcal{X}_{k-1}}\left(3 m_{k-1}\right) \\
\rightarrow \Omega_{\mathcal{X}_{k} / \mathcal{X}_{k-1}} \otimes \mathcal{O}_{\mathcal{X}_{k}}(2) \otimes \pi_{k-1, k}^{\star} \mathcal{O}_{\mathcal{X}_{k-1}}\left(3 m_{k-1}\right) \rightarrow 0
\end{aligned}
$$

setting $\underline{m_{k}}:=\left(3 m_{k-1}, 2\right)=2\left(\underline{m_{k-1}}, 1\right)+\left(\underline{m_{k-1}}, 0\right)$, that $\mathcal{O}_{\mathcal{X}_{k}}\left(\underline{m_{k}}\right)$ and $\mathcal{O}_{\mathcal{X}_{k+1}}\left(\underline{m_{k}}, 1\right)$ are nef. We find that the line bundle

$$
\mathcal{L}_{k}:=\mathcal{O}_{\mathcal{X}_{k}}\left(0,2 \cdot 3^{k-1} ; 2 \cdot 3^{k-2}, \ldots, 2 \cdot 3,2,1\right)
$$

is nef of total degree $3^{k}$. 


\section{Construction of differential equations}

Recall the setting of a family of hypersurfaces of $\mathbb{P}^{n+1}$ cut out in $C \times \mathbb{P}^{n+1}$ by a section of the line bundle $L$ :

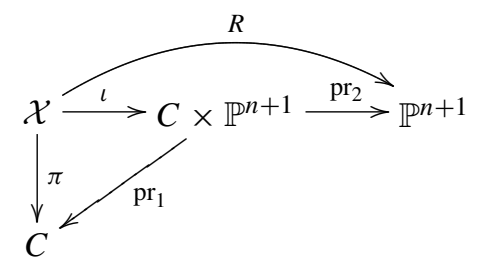

\subsection{Definitions of Segre classes}

Recall that the total Segre class $\operatorname{seg}(E)$ of a complex vector bundle $E \rightarrow X$ of rank $e$ is defined in the following way: its component $\operatorname{seg}_{i}(E)$ of degree $2 i$ is computed as $p_{\star} c_{1}\left(\mathcal{O}_{E}(1)\right)^{e-1+i}$, where $p: \mathbb{P}(E) \rightarrow X$ is the variety of rank one quotients of $E$. From this construction, one deduces that for a line bundle $L \rightarrow X$,

$$
\operatorname{seg}_{i}(E \otimes L)=\sum_{j=0}^{i}\left(\begin{array}{c}
e-1+i \\
i-j
\end{array}\right) \operatorname{seg}_{j}(E) c_{1}(L)^{i-j} .
$$

From the Grothendieck defining relation for Chern classes,

$$
c_{e}\left(p^{\star} E^{\star} \otimes \mathcal{O}_{E}(1)\right)=\sum_{i=0}^{e} p^{\star} c_{i}\left(E^{\star}\right) c_{1}\left(\mathcal{O}_{E}(1)\right)^{e-i}=0
$$

one infers that the total Segre class $\operatorname{seg}(E)$ is the formal inverse $c\left(E^{\star}\right)^{-1}$ of the total Chern class of the dual bundle $E^{\star}$. It is therefore multiplicative in short exact sequences.

\subsection{Computations on $\mathcal{X}$}

Write on $C \times \mathbb{P}^{n+1}$

$$
c_{1}(L)=d \operatorname{pr}_{2}^{\star} c_{1}\left(\mathcal{O}_{\mathbb{P}^{n+1}}(1)\right)+r \operatorname{pr}_{1}^{\star} c_{1}\left(\mathcal{O}_{C}(1)\right) .
$$

We have the relations $c_{1}\left(\mathcal{O}_{\mathbb{P}^{n+1}}(1)\right)^{n+2}=0, c_{1}\left(\mathcal{O}_{C}(1)\right)^{2}=0, c_{1}\left(\mathcal{O}_{\mathbb{P}^{n+1}}(1)\right)^{n+1}$. $c_{1}\left(\mathcal{O}_{C}(1)\right)=1$. Set on $\mathcal{X}$,

$$
\alpha:=R^{\star} c_{1}\left(\mathcal{O}_{\mathbb{P}^{n+1}}(1)\right) \quad \text { and } \quad \beta:=\pi^{\star} c_{1}\left(\mathcal{O}_{B}(1)\right) .
$$

Using $\alpha=\iota^{\star} \operatorname{pr}_{2}^{\star} c_{1}\left(\mathcal{O}_{\mathbb{P}^{n+1}}(1)\right)$ and $\beta=\iota^{\star} \operatorname{pr}_{1}^{\star} c_{1}\left(\mathcal{O}_{C}(1)\right)$, we find the relations

$$
\begin{aligned}
\alpha^{n+1} & =c_{1}(L) \cdot \operatorname{pr}_{2}^{\star} c_{1}\left(\mathcal{O}_{\mathbb{P}^{n+1}}(1)\right)^{n+1}=r, \\
\alpha^{n} \beta & =c_{1}(L) \cdot \operatorname{pr}_{2}^{\star} c_{1}\left(\mathcal{O}_{\mathbb{P}^{n+1}}(1)\right)^{n} \cdot \operatorname{pr}_{1}^{\star} c_{1}\left(\mathcal{O}_{C}(1)\right)=d .
\end{aligned}
$$

Hence, $r$ is the degree of the map $R:=\operatorname{pr}_{2} \circ \iota$, and $d$ is the degree of the generic member of the family $X_{c} \subset \mathbb{P}^{n+1}$. From the sequence (4.1) and the Euler sequence on $\mathbb{P}^{n+1}$, we 
infer that the total Segre class of $\mathcal{F}_{0}=\Omega_{\mathcal{X}}$ is

$$
\begin{aligned}
\operatorname{seg}\left(\mathcal{F}_{0}\right) & =\pi^{\star} \operatorname{seg}\left(\Omega_{B}\right) R^{\star} \operatorname{seg}\left(\Omega_{\mathbb{P}^{3}}\right) \iota^{\star} \operatorname{seg}\left(L_{0}^{\star}\right)^{-1} \\
& =\pi^{\star} \operatorname{seg}\left(\Omega_{B}\right) R^{\star} \operatorname{seg}\left(\mathbb{C}^{n+2} \otimes \mathcal{O}_{\mathbb{P}^{n+1}}(-1)\right) \iota^{\star} c\left(L_{0}\right) \\
& =\pi^{\star} \operatorname{seg}\left(\Omega_{B}\right) R^{\star} c\left(\mathcal{O}_{\mathbb{P}^{n+1}}(1)\right)^{-(n+2)} \iota^{\star} c\left(L_{0}\right) \\
& =(1+\chi \beta)(1+\alpha)^{-(n+2)}(1+d \alpha+r \beta) .
\end{aligned}
$$

We find that the Segre classes of $\mathcal{F}_{0}$ are polynomials in $(\alpha, \beta)$ with coefficients that are linear in $(r, d)$. In particular,

$$
\operatorname{seg}_{1}\left(\mathcal{F}_{0}\right)=(d-n-2) \alpha+(r+\chi) \beta .
$$

\subsection{A recursion formula}

Recall the defining relation for the bundles $\mathcal{F}_{k}$ on $\mathcal{X}_{k}$,

$$
0 \rightarrow \mathcal{O}_{\mathcal{X}_{k}}(1) \rightarrow \mathcal{F}_{k} \rightarrow \Omega_{\mathcal{X}_{k} / \mathcal{X}_{k-1}} \rightarrow 0,
$$

still valid for $k=0$ if we set $\mathcal{X}_{-1}=C, \mathcal{X}_{0}=\mathcal{X}, \mathcal{F}_{0}=\Omega_{\mathcal{X}}, \mathcal{O}_{\mathcal{X}}(1)=\pi^{\star} \Omega_{C}$, that is,

$$
0 \rightarrow \pi^{\star} \Omega_{C} \rightarrow \Omega_{\mathcal{X}} \rightarrow \Omega_{\mathcal{X} / C} \rightarrow 0 .
$$

We will also need the relative Euler sequence on $\mathcal{X}_{k}$,

$$
0 \rightarrow \Omega_{\mathcal{X}_{k} / \mathcal{X}_{k-1}} \rightarrow \pi_{k-1, k}^{\star} \mathcal{F}_{k-1} \otimes \mathcal{O}_{\mathcal{X}_{k}}(-1) \rightarrow \mathcal{O}_{\mathcal{X}_{k}} \rightarrow 0 .
$$

From the previous two sequences, we can compute the total Segre class of $\mathcal{F}_{k}$ in terms of that of $\mathcal{F}_{k-1}(k \geq 1)$. Set

$$
\alpha_{k}:=c_{1}\left(\mathcal{O}_{\mathcal{F}_{k-1}}(1)\right)=c_{1}\left(\mathcal{O}_{\mathcal{X}_{k}}(1)\right) .
$$

To begin with, the first Segre classes are easy to compute. We find

$$
\operatorname{seg}_{1}\left(\mathcal{F}_{k}\right)=\pi_{0, k}^{\star} \operatorname{seg}_{1}\left(\mathcal{F}_{0}\right)-n\left(\alpha_{k}+\pi_{k-1, k}^{\star} \alpha_{k-1}+\cdots+\pi_{1, k}^{\star} \alpha_{1}\right) .
$$

In general,

$$
\begin{aligned}
\operatorname{seg}\left(\mathcal{F}_{k}\right) & =\operatorname{seg}\left(\mathcal{O}_{\mathcal{X}_{k}}(1)\right) \operatorname{seg}\left(\Omega_{\mathcal{X}_{k} / \mathcal{X}_{k-1}}\right)=\operatorname{seg}\left(\mathcal{O}_{\mathcal{X}_{k}}(1)\right) \operatorname{seg}\left(\pi_{k-1, k}^{\star} \mathcal{F}_{k-1} \otimes \mathcal{O}_{\mathcal{X}_{k}}(-1)\right) \\
& =\sum_{\ell=0}^{(k+1) n+1} \sum_{i=0}^{\ell} \operatorname{seg}_{\ell-i}\left(\mathcal{O}_{\mathcal{X}_{k}}(1)\right) \operatorname{seg}_{i}\left(\Omega_{\mathcal{X}_{k} / \mathcal{X}_{k-1}}\right) \\
& =\sum_{\ell=0}^{(k+1) n+1} \sum_{i=0}^{\ell} \operatorname{seg}_{\ell-i}\left(\mathcal{O}_{\mathcal{X}_{k}}(1)\right) \operatorname{seg}_{i}\left(\pi_{k-1, k}^{\star} \mathcal{F}_{k-1} \otimes \mathcal{O}_{\mathcal{X}_{k}}(-1)\right) \\
& =\sum_{\ell=0}^{(k+1) n+1} \sum_{i=0}^{\ell} \alpha_{k}^{\ell-i} \sum_{j=0}^{i}\left(\begin{array}{c}
n+i \\
i-j
\end{array}\right) \pi_{k-1, k}^{\star} \operatorname{seg}_{j}\left(\mathcal{F}_{k-1}\right)\left(-\alpha_{k}\right)^{i-j}
\end{aligned}
$$




$$
\begin{aligned}
& =\sum_{\ell=0}^{(k+1) n+1} \sum_{j=0}^{\ell} \pi_{k-1, k}^{\star} \operatorname{seg}_{j}\left(\mathcal{F}_{k-1}\right) \alpha_{k}^{\ell-j} \sum_{i=j}^{\ell}(-1)^{i-j}\left(\begin{array}{c}
n+i \\
i-j
\end{array}\right) \\
& =\sum_{\ell=0}^{(k+1) n+1} \sum_{j=0}^{\ell}\left[\sum_{i=0}^{\ell-j}(-1)^{i}\left(\begin{array}{c}
n+j+i \\
i
\end{array}\right)\right] \pi_{k-1, k}^{\star} \operatorname{seg}_{j}\left(\mathcal{F}_{k-1}\right) \alpha_{k}^{\ell-j} .
\end{aligned}
$$

Defining the numbers $\mathbb{L}_{e}^{f+e}:=\sum_{i=0}^{f}(-1)^{i}\left(\begin{array}{c}e+i \\ e\end{array}\right)$, we get

$$
\operatorname{seg}_{\ell}\left(\mathcal{F}_{k}\right)=\sum_{a+b=\ell} \mathbb{L}_{n+a}^{n+\ell} \pi_{k-1, k}^{\star} \operatorname{seg}_{a}\left(\mathcal{F}_{k-1}\right) \alpha_{k}^{b} .
$$

\subsection{Estimates for intersection numbers}

The idea comes from [Div-09]. Recall that the line bundle $\mathcal{L}_{k}:=\mathcal{O}_{\mathcal{X}_{k}}\left(0,2 \cdot 3^{k-2}\right.$; $\left.2 \cdot 3^{k-1}, \ldots, 2 \cdot 3,2,1\right)$ is nef on $\mathcal{X}_{k}$. Note that its entries depend neither on the degree $d$ nor on the variation $r$. Its first Chern class is

$$
l_{k}:=\alpha_{k}+2 \pi_{k-1, k}^{\star} \alpha_{k-1}+6 \pi_{k-2, k}^{\star} \alpha_{k-2}+\cdots+2 \cdot 3^{k-2} \pi_{1, k}^{\star} \alpha_{1}+2 \cdot 3^{k-1} \pi_{0, k}^{\star} \alpha .
$$

We are in a position to prove

Lemma 5.1. For $r, d \gg 1$,

$$
\begin{aligned}
\left(\pi_{k-1, k}\right)_{\star} l_{k}^{n+1} & \geq \pi_{0, k-1}^{\star} \operatorname{seg}_{1}\left(\mathcal{F}_{0}\right), \\
\operatorname{seg}_{1}\left(\mathcal{F}_{0}\right)^{n+1} & \sim(n+2) r d^{n+1}, \\
l_{1}^{m_{1}} \cdots l_{s}^{m_{s}} \cdot \alpha & \leq A_{n+1}(r, d), \\
l_{1}^{m_{1}} \cdots l_{s}^{m_{s}} \cdot \beta & \leq B_{n+1}(r, d),
\end{aligned}
$$

where $A_{n+1}$ and $B_{n+1}$ are polynomials in $(r, d)$ of degree less than or equal to $n+1$.

The output is that the leading numerical term comes from the relative canonical degree.

Proof. (1) Recall from (5.4) that

$$
\begin{aligned}
\left(\pi_{k-1, k}\right)_{\star} & l_{k}^{n+1} \\
= & \operatorname{seg}_{1}\left(\mathcal{F}_{k-1}\right)+(n+1)\left(2 \alpha_{k-1}+6 \alpha_{k-2}+\cdots+2 \cdot 3^{k-2} \alpha_{1}+2 \cdot 3^{k-1} \alpha\right) \\
= & \pi_{0, k-1}^{\star} \operatorname{seg}_{1}\left(\mathcal{F}_{0}\right)-n\left(\alpha_{k-1}+\alpha_{k-2}+\cdots+\alpha_{1}\right) \\
& +(n+1)\left(2 \alpha_{k-1}+6 \alpha_{k-2}+\cdots+2 \cdot 3^{k-2} \alpha_{1}+2 \cdot 3^{k-1} \alpha\right) \\
= & \pi_{0, k-1}^{\star} \operatorname{seg}_{1}\left(\mathcal{F}_{0}\right)+(n+2) \alpha_{k-1}+(5 n+6) \alpha_{k-2}+\cdots \\
& +\left((n+1) 2 \cdot 3^{j-1}-n\right) \alpha_{k-j}+\left((n+1) 2 \cdot 3^{j}-n\right) \alpha_{k-j-1}+\cdots \\
& +\left((n+1) 2 \cdot 3^{k-2}-n\right) \alpha_{1}+2 \cdot 3^{k-1} \alpha
\end{aligned}
$$

The claim follows from the inequalities $(n+1) 2 \cdot 3^{j}-n \geq 3\left[(n+1) 2 \cdot 3^{j-1}-n\right]$ that ensure the nefness of $\left(\pi_{k-1, k}\right)_{\star} l_{k}^{n+1}-\pi_{0, k-1}^{\star} \operatorname{seg}_{1}\left(\mathcal{F}_{0}\right)$. 
(2) Just compute

$$
\begin{aligned}
\operatorname{seg}_{1}\left(\mathcal{F}_{0}\right)^{n+1} & =((d-n-2) \alpha+(r+\chi) \beta)^{n+1} \\
& \sim d^{n+1} \alpha^{n+1}+(n+1) r d^{n} \alpha^{n} \beta=(n+2) r d^{n+1}
\end{aligned}
$$

(3) It follows from the recursion formula that, computed in $\mathcal{X}$ of dimension $n+1$,

$$
l_{1}^{m_{1}} \cdots l_{s}^{m_{s}} \cdot \alpha=\sum_{k \leq n} \sum_{k \leq n} \sum_{\substack{I=\left(i_{1}, \cdots, i_{k}\right) \\ 1 \leq i_{1} \cdots \leq i_{k}}} C_{I} s_{i_{1}}\left(\mathcal{F}_{0}\right) \cdots s_{i_{k}}\left(\mathcal{F}_{0}\right) \cdot \alpha .
$$

Recall that the Segre classes of $\mathcal{F}_{0}$ are polynomials in $(\alpha, \beta)$ whose coefficients are linear in $(r, d)$ and use $(5.1)$ to get

$$
l_{1}^{m_{1}} \cdots l_{s}^{m_{s}} \cdot \alpha=P(r, d) \alpha^{n+1}+Q(r, d) \alpha^{n} \beta=P(r, d) r+Q(r, d) d
$$

where $P$ and $Q$ are polynomials in $(r, d)$ of degree less than or equal to $n$.

(4) In the previous computations, the class $\alpha$ can be replaced by the class $\beta$ to get

$$
l_{1}^{m_{1}} \ldots l_{s}^{m_{s}} \cdot \beta=R(r, d) \alpha^{n} \beta=R(r, d) d
$$

where $R$ is a polynomial in $(r, d)$ of degree less than or equal to $n$.

\subsection{Choice of line bundles}

Fix $\rho: C_{\rho} \rightarrow C$. We choose to work on the jet space of order $\kappa=n+1$. We define on $\mathcal{X}_{n+1}$ a line bundle that is the tensor product of the nef line bundles constructed in Section 4:

$$
A=\mathcal{L}_{n+1} \otimes \cdots \otimes \mathcal{L}_{1}
$$

and we choose $B$ so that $L:=A-B$ has negative component along $\operatorname{Pic}(\mathcal{X} / C)$ and satisfies the vanishing criterion, that is, for some fixed positive rational number $x$, and some positive rational number $y$ with

$$
\frac{\chi_{\rho}}{\operatorname{deg} \rho} \sum_{j=1}^{n+1}\left(3^{j}-2 \cdot 3^{j-1}\right)=\frac{\chi_{\rho}}{\operatorname{deg} \rho} \frac{3^{n+1}-1}{2}<y,
$$

we have

$$
B=\mathcal{O}_{\mathbb{P}^{n+1}}\left(2 \cdot 3^{n+1-1}+\cdots+2+x\right) \otimes \mathcal{O}_{C}(y)=\mathcal{O}_{\mathbb{P}^{n+1}}\left(3^{n+1}-1+x\right) \otimes \mathcal{O}_{C}(y)
$$

where $\mathcal{O}_{C}(y)$ is any line bundle on the curve $C$ of degree $y$. The precise knowledge of the nef cone of $\mathcal{X}$ as in Proposition 4.2 could improve, from the effectivity point of view, the choice of $A$ and $B$. 
With $\kappa=n+1$, we have $\operatorname{dim} \mathcal{X}_{\kappa}=\kappa(n+1)$. Hence, if we only omit intersections of nef classes,

$$
\begin{aligned}
A^{\operatorname{dim} \mathcal{X}_{\kappa}} & =\left(l_{\kappa}+\cdots+l_{1}\right)^{\operatorname{dim} \mathcal{X}_{\kappa}} \geq l_{\kappa}^{(n+1)} \cdots l_{1}^{(n+1)} \\
& \geq \pi_{0, \kappa-1}^{\star} \operatorname{seg}_{1}\left(\mathcal{F}_{0}\right) \cdot l_{\kappa-1}^{(n+1)} \cdots l_{1}^{(n+1)} \geq \pi_{0, \kappa-2}^{\star} \operatorname{seg}_{1}\left(\mathcal{F}_{0}\right)^{2} \cdot l_{\kappa-2}^{(n+1)} \cdots l_{1}^{(n+1)} \\
& \cdots \\
& \geq \operatorname{seg}_{1}\left(\mathcal{F}_{0}\right)^{\kappa} \sim(n+2) r d^{n+1}
\end{aligned}
$$

thanks to Lemma 5.1. On the other hand, by the same lemma, $A^{\operatorname{dim} \mathcal{X}_{k}-1} \cdot B$ is bounded above by a polynomial in $(r, d)$ of degree less than or equal to $n+1$. If $r$ and $d$ are chosen large enough, we find that $A^{\operatorname{dim} \mathcal{X}_{\kappa}}-\operatorname{dim} \mathcal{X}_{k} A^{\operatorname{dim} \mathcal{X}_{k}-1} \cdot B$ is positive. Hence, the line bundle $A-B$ is big and the sections of its powers provide non-zero equations for the jets of sections of the family $\mathcal{X}_{\rho} \rightarrow C_{\rho}$.

We have proved a precise version of Theorem 1.

Theorem 2. Fix $\rho: C_{\rho} \rightarrow C$. Fix a positive rational $x$ and a large enough positive rational $y$. For $r, d \gg 1$, for a general family $\pi$ of hypersurfaces of $\mathbb{P}^{n+1}$ of degree $d$ and variation $r$, there is a non-trivial differential equation of order $n+1$ and total weight $\underline{m}$, given by a section of the line bundle

$$
\mathcal{O}_{\mathcal{X}_{n+1}}(\underline{m}) \otimes \mathcal{O}_{\mathbb{P}^{n+1}}(-|\underline{m}| x) \otimes \mathcal{O}_{C}(-|\underline{m}| y),
$$

satisfied by all sections of the pull-back family $\pi_{\rho}$.

\subsection{Height inequalities}

We now look for a statement that incorporates the dependence on the ramified cover $C_{\rho} \rightarrow C$. We work on $\mathcal{X}_{n+1}$ with $A=\mathcal{L}_{n+1} \otimes \cdots \otimes \mathcal{L}_{1}$ and we choose $B$ so that $L:=A-B$ has negative component on $\operatorname{Pic}(\mathcal{X} / B)$, that is, $B=\mathcal{O}_{\mathbb{P}^{n+1}}\left(3^{n+1}-1+x\right)$. The previous computations show that $A-B$ is big for large enough $r$ and $d$. As a result, we obtain

Corollary 5.2. Fix a positive integer $x$. For $r, d \gg 1$, for a general family $\mathcal{X}$ of hypersurfaces in $\mathbb{P}^{n+1}$ of degree $d$ and variation $r$, there exists a proper algebraic set $\mathcal{Y} \subset \mathcal{X}_{n+1}$ such that for every finite ramified cover $\rho: C_{\rho} \rightarrow C$ and every section s of $\rho^{\star} \mathcal{X} \rightarrow C_{\rho}$ whose $(n+1)$-jet does not lie in $\rho^{\star} \mathcal{Y}$, the following height inequality holds:

$$
h(s(C)):=\frac{s\left(C_{\rho}\right) \cdot \mathcal{O}_{\mathbb{P}^{n+1}}(1)}{\operatorname{deg} \rho} \leq \frac{3^{n+1}-1}{2 x} \frac{\chi_{\rho}}{\operatorname{deg} \rho} .
$$

This is an analog of the first part of Vojta's result [Voj-78]. The deepest part, dealing with sections having $(n+1)$-jet inside $\mathcal{Y}$, would require an analog of Jouanoulou's result on foliations [Jou-78], which seems out of reach now. 


\section{Non-Zariski density}

We follow the ideas of Siu [Siu-04], described in detail in [D-M-R-08]. Let $d$ be a positive integer, $C$ be a connected compact complex smooth curve, and $\lambda$ be a holomorphic line bundle on $C$ of degree $r$. Consider the linear system $\mathbb{P}^{N}:=\left|\lambda \otimes \mathcal{O}_{\mathbb{P}^{n+1}}(d)\right|$ on $C \times \mathbb{P}^{n+1}$, every element $F$ of which represents a family $\pi^{F}: \mathcal{X}^{F} \rightarrow C$ of degree $d$ hypersurfaces in $\mathbb{P}^{n+1}$ parametrised by $C$ with variation $r$. Consider the associated universal family

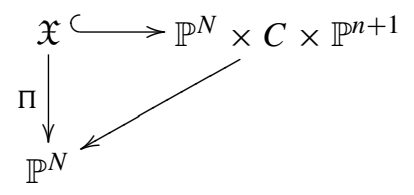

The variable $t$ will denote a coordinate on the curve $C$. Coordinates on $\mathbb{P}^{n+1}$ will be denoted by $z$ 's, while relative coordinates on $\mathcal{X}^{F} \rightarrow C$ will be denoted by $x$ 's. Constant sections are those whose 1 -jet lies inside $\left\{z_{1}^{\prime}=\cdots=z_{n+1}^{\prime}=0\right\}$. We will denote by $\mathfrak{X}_{\kappa}$ the $\Pi$-relative $\kappa$-jet space of sections of the families $\left(\mathcal{X}^{F} \rightarrow C\right)_{F \in \mathbb{P}^{N}}$. It comes equipped with natural line bundles $\mathfrak{L}_{-\mu,-x, \underline{m}} \rightarrow \mathfrak{X}$ that restrict on $\mathcal{X}^{F}$ as $\mathcal{L}_{-\mu,-x, \underline{m}}^{F}$.

\subsection{Proof using vector fields on universal families}

Choose an integer $\kappa \geq n+1$. Fix positive rational numbers

$$
x>3^{n+1}-1+\kappa^{2}+2 \kappa \quad \text { and } \quad y>\frac{\chi \rho}{\operatorname{deg} \rho} \frac{3^{n+1}-1}{2} .
$$

Consider a family $\pi^{F}$. Define $\mu:=\mathcal{O}_{C}(y)$ to be any line bundle of degree $y$ on $C$. Select the line bundle on $\mathcal{X}_{\kappa}^{F}$,

$$
\mathcal{L}_{-\mu,-x, \underline{m}}^{F}:=\mathcal{O}_{\mathcal{X}_{\kappa}^{F}}(\underline{m}) \otimes \mathcal{O}_{\mathbb{P}^{n+1}}(-|\underline{m}| x) \otimes \mu^{-|\underline{m}|} .
$$

We assume that $r, d \gg 1$, and $\underline{m}$ is a weight provided by Theorem 2 so that $\mathcal{L}_{-\mu,-x, \underline{m}}^{F}$ is big and effective and satisfies the vanishing criterion. The push-forward $\left(\pi_{\kappa, 0}^{F}\right)_{\star} \sigma$ of a non-zero section $\sigma$ of $\mathcal{L}_{-\mu,-x, \underline{m}}^{F}$ is a non-zero section of the vector bundle $\left(\pi_{\kappa, 0}^{F}\right)_{\star} \mathcal{L}_{-\mu,-x, \underline{m}}^{F} \rightarrow \mathcal{X}^{F}$. We can now prove the precise version of the main theorem.

Theorem 3. If $r, d$ are large enough, if the equation $F$ is general in $\mathbb{P}^{N}$, and if $|\underline{m}|$ is large enough, then there is a non-zero section $\sigma$ of the line bundle $\mathcal{L}_{-\mu,-x, \underline{m}}^{F}$ defined above such that every non-constant section s of $\pi^{F}$ satisfies

$$
s(C) \subset \operatorname{Zero}\left(\left(\pi_{\kappa, 0}^{F}\right)_{\star} \sigma\right) \subset \mathcal{X}^{F} .
$$

The point is that the generality assumption on $F$ enables one to transfer the constraints on the jet $s_{k}$ given by the vanishing criterion into constraints on the section $s$ itself.

Proof of Theorem 3. We only sketch the proof, the details being close to those given in [D-M-R-08]. Aiming at a contradiction, assume that there exists a $c_{0}$ in $C$ where 
$\left(\pi_{\kappa, 0}^{F}\right)_{\star} \sigma\left(s\left(c_{0}\right)\right) \neq 0$. Now view the section $\sigma$ as a meromorphic function

$$
\mathcal{X}_{\kappa}^{F} \rightarrow \mathbb{C}, \quad \zeta_{\kappa} \mapsto \sum_{w l(I)=m} q_{I}(t, x)\left(x^{\prime}\left(\zeta_{\kappa}\right)\right)^{i_{1}} \cdots\left(x^{(\kappa)}\left(\zeta_{\kappa}\right)\right)^{i_{\kappa}},
$$

where $I=\left(i_{1}, \ldots, i_{k}\right), w l(I)=\sum_{j=1}^{k} j i_{j}$ and the $q_{I}(t, x)$ are meromorphic functions on $\mathcal{X}^{F}$, holomorphic when viewed as local sections of $\mathcal{O}_{\mathbb{P}^{n+1}}(-|\underline{m}| \delta) \otimes \mu^{-|\underline{m}|}$. The assumption $\left(\pi_{\kappa, 0}^{F}\right)_{\star} \sigma\left(s\left(c_{0}\right)\right) \neq 0$ translates into the existence of a multiindex $I_{0}$ of weighted length $m$ such that $q_{I_{0}}\left(s_{\kappa}\left(c_{0}\right)\right) \neq 0$.

We now use the semicontinuity theorem: for each multiindex $\underline{M}$, the set of parameters $G$ in $\mathbb{P}^{N}$ such that the line bundle $\mathcal{L}_{-\mu,-x, \underline{M}}^{G}$ has a section is an algebraic subset of $\mathbb{P}^{N}$. The countable union of those contains the complement of a proper algebraic subset (parametrising singular total spaces $\mathcal{X}^{F}$ ). Hence there is a multiindex $\underline{m}$ valid for general hypersurfaces. The semicontinuity theorem again ensures that the rank of the corresponding direct image is positive. By the generality assumption on $F$, we may extend the section $\left(\pi_{\kappa, 0}^{F}\right)_{\star} \sigma$ to a section $\left(\Pi_{\kappa, 0}\right)_{\star} \widetilde{\sigma}$ of $\left(\Pi_{\kappa, 0}\right)_{\star} \mathfrak{L}_{-\mu,-x, \underline{m}} \rightarrow \mathfrak{X}$ on a neighbourhood $U^{F}$ of $\mathcal{X}^{F}$ in $\mathfrak{X}$.

We now need vector fields to isolate the coefficient $q_{I_{0}}$ by repeated derivations.

Proposition 6.1. Every vector in

$$
T\left(\mathcal{X}_{\kappa}^{F} / \mathcal{X}^{F}\right)_{\left(s_{\kappa}\left(c_{0}\right)\right)} \subset\left(T \mathcal{X}_{\kappa}^{F}\right)_{\left(s_{\kappa}\left(c_{0}\right)\right)}=T\left(\mathfrak{X}_{\kappa} / \mathbb{P}^{N}\right)_{\left(F, s_{\kappa}\left(c_{0}\right)\right)} \subset\left(T \mathfrak{X}_{\kappa}\right)_{\left(F, s_{\kappa}\left(c_{0}\right)\right)}
$$

outside the set $\Pi_{\kappa, 1}^{-1}\left\{z_{1}^{\prime}=\cdots=z_{n+1}^{\prime}=0\right\}$ is the value of a meromorphic vector field on $\Pi_{\kappa, 0}^{-1}\left(U^{F}\right) \subset \mathfrak{X}_{\kappa}$ holomorphic when viewed with values in $\Pi_{\kappa, 0}^{\star} \mathcal{O}_{\mathbb{P}^{n+1}}\left(\kappa^{2}+2 \kappa\right)$.

Take it for granted until the next subsection. When we differentiate the extended meromorphic function $\widetilde{\sigma}$ with the meromorphic vector fields obtained at most $|\underline{m}|$ times and restrict to the fibre over $F$, we get meromorphic functions on $\mathcal{X}^{F}$ that in turn can be viewed as a section of $\mathcal{L}_{-\mu,-x+\left(\kappa^{2}+2 \kappa\right), m}^{F}$. Recall that $-x+3^{n+1}-1+\left(\kappa^{2}+2 \kappa\right)$ is still negative, so that $s_{\kappa}$ still has to satisfy this new equation. If we choose the vector fields in a suitable way, according to Proposition 6.1, this contradicts $q_{I_{0}}\left(s_{\kappa}\left(c_{0}\right)\right) \neq 0$.

The proof of the main theorem is now finished as follows. If the images of constant sections dominated the total space $\mathcal{X}^{F}$, then because they have bounded height (they are constant in the product $C \times \mathbb{P}^{n+1}$ ), there would exist an algebraic set $S$, a Hilbert scheme component of the space of sections, and a dominant map $C \times S \rightarrow \mathcal{X}^{F}$ over $C$. The criterion of birational splitting of Maehara and Moriwaki [Mor-94], which follows from positivity of direct images of pluricanonical line bundles, would show that the family has to be birationally trivial.

\subsection{Constructing vector fields on universal families}

In homogeneous coordinates, if we choose a basis for $\mathbb{C}^{n+2}$, the corresponding basis $\left(Z^{\alpha}\right)_{\alpha}$ of monomials for $\left|\mathcal{O}_{\mathbb{P}^{n+1}}(d)\right|$, and a basis $\left(\Phi_{\beta}\right)_{\beta}$ for $|\lambda|$, the hypersurface $\mathfrak{X}$ of 
$\mathbb{P}^{N} \times C \times \mathbb{P}^{n+1}$ is defined by the equation

$$
\sum_{\alpha, \beta} \mathfrak{A}_{\alpha}^{\beta} \Phi_{\beta} Z^{\alpha}=0
$$

On the open set $\left\{\mathfrak{A}_{0, d, 0, \ldots, 0}^{0} \neq 0\right\} \times\left\{\Phi_{0}(b) \neq 0\right\} \times\left\{Z^{0} \neq 0\right\}$ the equation can be rewritten in inhomogeneous coordinates as

$$
\mathfrak{F}=z_{1}^{d}+\sum_{\substack{\alpha \in \mathbb{N}^{n+1},|\alpha| \leq d \\ \alpha \neq(d, 0, \ldots, 0) \\ \beta \geq 1}} a_{\alpha}^{\beta} \varphi_{\beta}(b) z^{\alpha}=0 .
$$

Over this open set, the natural open set of the $\Pi$-relative $\kappa$-jet space $\mathfrak{X}_{\kappa}$ is given inside $\mathbb{C}^{N} \times U \times \mathbb{C}^{n+1} \times \underbrace{\mathbb{C}^{n+1} \times \cdots \times \mathbb{C}^{n+1}}_{\kappa \text { times }}$ in terms of the operator

$$
\mathfrak{D}:=\frac{\partial}{\partial t}+\sum_{\nu=0}^{\kappa} \sum_{j=1}^{n+1} z_{j}^{(v+1)} \frac{\partial}{\partial z_{j}^{(v)}}
$$

by the following set of equations:

$$
\begin{aligned}
\sum_{\substack{\alpha \in \mathbb{N}^{n+1} \\
|\alpha| \leq d \\
\beta \geq 1}} a_{\alpha}^{\beta} \varphi_{\beta}(t) z^{\alpha}=\mathfrak{D}\left(\sum a_{\alpha}^{\beta} \varphi_{\beta}(t) z^{\alpha}\right)=\mathfrak{D}^{2}\left(\sum a_{\alpha}^{\beta} \varphi_{\beta}(t) z^{\alpha}\right) \\
=\cdots=\mathfrak{D}^{\kappa}\left(\sum a_{\alpha}^{\beta} \varphi_{\beta}(t) z^{\alpha}\right)=0 .
\end{aligned}
$$

Those are the equations one infers by differentiating the relation $\sum a_{\alpha}^{\beta} \varphi_{\beta}(t) z^{\alpha}(t)=0$ satisfied by the sections $t \mapsto\left(t, z_{1}(t), \ldots, z_{n+1}(t)\right)$ of a family $\Pi^{-1}(F)$, after substituting $z_{j}^{(v)}:=\partial^{v} z_{j}(t) / \partial t^{\nu}$

Denote the partial sum $\sum_{\alpha \in \mathbb{N}^{n+1},|\alpha| \leq d} a_{\alpha}^{\beta} z^{\alpha}$ by $\mathfrak{F}_{\beta}$. The equations for a vector field $T$ of the special shape $T:=\left(\sum_{\beta} T_{\beta}\right)+T_{z}$, where

$$
T_{\beta}:=\sum_{\alpha} A_{\alpha}^{\beta} \frac{\partial}{\partial a_{\alpha}^{\beta}} \quad \text { and } q \quad T_{z}:=\sum_{\nu=0}^{\kappa} \sum_{j=1}^{n+1} P_{j}^{\nu} \frac{\partial}{\partial z_{j}^{(\nu)}},
$$

to be tangent to $\mathfrak{X}_{\kappa}$ can be rewritten, thanks to the Leibniz formula and the fact that when $\beta \neq \gamma, T_{\beta} \cdot \mathfrak{D}^{a} \mathfrak{F}_{\gamma}=0$, in terms of the operator

$$
D:=\sum_{v=0}^{\kappa} \sum_{j=1}^{n+1} z_{j}^{(v+1)} \frac{\partial}{\partial z_{j}^{(v)}}
$$


as

$$
\begin{array}{r}
\sum_{\beta \geq 1} \varphi_{\beta}(t)\left(T_{\beta}+T_{z}\right) \cdot \mathfrak{F}_{\beta}=0, \\
\sum_{\beta \geq 1} \varphi_{\beta}(t)\left(T_{\beta}+T_{z}\right) \cdot D\left(\mathcal{F}_{\beta}\right)+\varphi_{\beta}^{\prime}(t)\left(T_{\beta}+T_{z}\right) \cdot \mathfrak{F}_{\beta}=0, \\
\sum_{\beta \geq 1} \varphi_{\beta}(t)\left(T_{\beta}+T_{z}\right) \cdot D^{2}\left(\mathcal{F}_{\beta}\right)+2 \varphi_{\beta}^{\prime}(t)\left(T_{\beta}+T_{z}\right) \cdot D\left(\mathcal{F}_{\beta}\right)+\varphi_{\beta}^{\prime \prime}(t)\left(T_{\beta}+T_{z}\right) \cdot \mathfrak{F}_{\beta}=0, \\
\vdots \\
\sum_{\beta \geq 1} \sum_{a=0}^{\kappa}\left(\begin{array}{c}
\kappa \\
a
\end{array}\right) \varphi_{\beta}^{(\kappa-a)}\left(T_{\beta}+T_{z}\right) \cdot D^{a}\left(\mathfrak{F}_{\beta}\right)=0 .
\end{array}
$$

A set of sufficient conditions is therefore

$$
\forall \beta, \quad\left(T_{\beta}+T_{z}\right) \cdot \mathfrak{F}_{\beta}=\left(T_{\beta}+T_{z}\right) \cdot D\left(\mathfrak{F}_{\beta}\right)=\cdots=\left(T_{\beta}+T_{z}\right) \cdot D^{\kappa}\left(\mathfrak{F}_{\beta}\right)=0
$$

reducing to the absolute case. Note however that, as Theorem 2 provides a differential equation of order $n+1$, we need to consider $(n+1)$-jets of hypersurfaces in $\mathbb{P}^{n+1}$, whereas the by now well settled results are for $n$-jets.

\subsection{Constructing vector fields in the absolute case}

We follow the ideas of Siu [Siu-04], Păun [Păun-08], Rousseau [Rou-07] and Merker [Mer-09]. For notational simplicity, we will replace $\beta$ by a dot in the following. The exponents in brackets will be relative to the absolute operator $D$.

Write $\left(T .+T_{z}\right) \cdot D^{l+1}(\mathfrak{F})=.\left[T .+T_{z}, D\right] D^{l}(\mathfrak{F})+.D\left(\left(T .+T_{z}\right) \cdot D^{l}(\mathfrak{F}).\right)$ to infer that a set of sufficient conditions for the special vector field $T$. $+T_{z}$ to contribute to a tangent to $\mathfrak{X}_{\kappa}$ is

$$
\begin{aligned}
\left(T .+T_{z}\right) \cdot \mathfrak{F} .=\left[T .+T_{z}, D\right] \cdot \mathfrak{F} .=[T .+ & \left.T_{z}, D\right] \cdot D(\mathfrak{F} .) \\
& =\cdots=\left[T .+T_{z}, D\right] \cdot D^{\kappa-1}(\mathfrak{F} .)=0 .
\end{aligned}
$$

We will now further restrict the shape of the chosen vector field to simplify its commutator with $D$.

Lemma 6.2. Let $A_{\alpha}$ and $P$ be functions in the $\left(z_{i}^{(v)}\right)$ variables. The commutator of the very special vector field

$$
T+T_{z}=\sum_{\alpha} A_{\alpha}^{\cdot} \frac{\partial}{\partial a_{\alpha}^{\cdot}}+\sum_{\nu=0}^{\kappa} P^{(v)} \frac{\partial}{\partial z_{j}^{(v)}}
$$

with $D$ is

$$
\left[T+T_{z}, D\right]=-\sum_{\alpha}\left(A_{\alpha}^{*}\right)^{\prime} \frac{\partial}{\partial a_{\alpha}}-P^{(\kappa+1)} \frac{\partial}{\partial z_{j}^{(\kappa)}}
$$


Proof. Simply check that

$$
\begin{array}{rlrl}
T .(D) & =0, & D(T .) & =\sum_{\alpha}\left(A_{\alpha}^{\cdot}\right)^{\prime} \frac{\partial}{\partial a_{\alpha}^{\prime}}, \\
T_{z}(D) & =\sum_{\nu=1}^{\kappa} P^{(\nu)} \frac{\partial}{\partial z_{j}^{(\nu-1)}}=\sum_{\nu=0}^{\kappa-1} P^{(\nu+1)} \frac{\partial}{\partial z_{j}^{(v)}}, & D\left(T_{z}\right)=\sum_{\nu=0}^{\kappa} P^{(v+1)} \frac{\partial}{\partial z_{j}^{(v)}} .
\end{array}
$$

We infer that a set of sufficient conditions for the very special vector field $T .+T_{z}$ to contribute to a tangent vector field to $\mathfrak{X}_{\kappa}$ is

$$
\begin{aligned}
& \sum_{\alpha} A_{\alpha} z^{\alpha}+P \sum_{\alpha} a_{\alpha} \frac{\partial z^{\alpha}}{\partial z_{j}}=0 \\
& -\sum_{\alpha}\left(A_{\alpha}^{\prime}\right)^{\prime} z^{\alpha}=-\sum_{\alpha}\left(A_{\alpha}^{\prime}\right)^{\prime}\left(z^{\alpha}\right)^{\prime}=-\sum_{\alpha}\left(A_{\alpha}^{*}\right)^{\prime}\left(z^{\alpha}\right)^{(2)} \\
& =\cdots=-\sum_{\alpha}\left(A_{\alpha}^{*}\right)^{\prime}\left(z^{\alpha}\right)^{(\kappa-1)}=0
\end{aligned}
$$

or equivalently, using the formula $D^{l+1}\left(\sum_{\alpha} A_{\alpha} z^{\alpha}\right)=\sum_{\alpha} A_{\alpha}\left(z^{\alpha}\right)^{(l+1)}+\sum_{\alpha} A_{\alpha}^{\prime}\left(z^{\alpha}\right)^{(l)}+$ $\sum_{k=0}^{l-1} D^{l-k}\left(\sum_{\alpha} A_{\alpha}^{\prime}\left(z^{\alpha}\right)^{(k)}\right)$,

$$
\begin{aligned}
\sum_{\alpha} A_{\alpha} z^{\alpha} & =-P \sum_{\alpha} a_{\alpha} \frac{\partial z^{\alpha}}{\partial z_{j}} \\
\sum_{\alpha} A_{\alpha}\left(z^{\alpha}\right)^{\prime} & =\left(-P \sum_{\alpha} a_{\alpha} \frac{\partial z^{\alpha}}{\partial z_{j}}\right)^{\prime} \\
& \vdots \\
\sum_{\alpha} A_{\alpha}\left(z^{\alpha}\right)^{(\kappa)} & =\left(-P \sum_{\alpha} a_{\alpha} \frac{\partial z^{\alpha}}{\partial z_{j}}\right)^{(\kappa)}
\end{aligned}
$$

or also

$$
\begin{aligned}
& \sum_{\alpha} A_{\alpha} z^{\alpha}=-P \sum_{\alpha} a_{\alpha} \frac{\partial z^{\alpha}}{\partial z_{j}}, \\
& \sum_{\alpha}\left(A_{\alpha}^{\cdot}\right)^{\prime} z^{\alpha}=\sum_{\alpha}\left(A_{\alpha}^{\cdot}\right)^{\prime \prime} z^{\alpha}=\sum_{\alpha}\left(A_{\alpha}^{\cdot}\right)^{(3)} z^{\alpha}=\cdots=\sum_{\alpha}\left(A_{\alpha}^{\cdot}\right)^{(\kappa)} z^{\alpha}=0 .
\end{aligned}
$$

When $P$ is of degree less than 2, the first equation in (6.2) can be satisfied with constant $A_{\alpha}$, making the other equations tautological.

When $P$ is of the form $P=z_{i}^{k}$, because the only non-zero term in the right hand side of (6.2) can be written as

$$
\sum_{|\beta| \leq d} b_{\beta} z^{\beta}+\sum_{\ell=1}^{k-1} \sum_{|\beta|=d} b_{\beta}^{\cdot \ell} z^{\beta+\ell \epsilon_{i}},
$$


we look for $A_{\alpha}$ in the form

$$
A_{\alpha}:=\sum_{\substack{\gamma,|\gamma| \leq \kappa \\|\alpha+\gamma| \leq d}} A_{\alpha}^{\cdot \gamma} z^{\gamma}+\sum_{\ell=1}^{\min \left(\alpha_{i}, k-1\right)} \sum_{\substack{\gamma,|\gamma| \leq \kappa \\\left|\alpha+\gamma-\ell \epsilon_{i}\right|=d}} A_{\alpha}^{\cdot \ell, \gamma} z^{\gamma} .
$$

Note that as $\alpha_{i} \geq \ell$, the multiindex $\alpha+\gamma-\ell \epsilon_{i}$ is non-negative. Then (6.2) can be rewritten, after recursive simplifications of all terms involving a $z_{l}^{(k)}$-variable with $k>1$, as a set of systems, one for each multiindex $\mu+\ell \epsilon_{i}$ where $\mu$ is a multiindex of length $|\mu| \leq d$ when $\ell=0$, or $|\mu|=d$ when $1 \leq \ell \leq k-1$. They have disjoint sets of indeterminates $\left(A_{\alpha}^{\cdot \ell, \gamma}\right)_{\alpha+\gamma=\mu+\ell \epsilon_{i},|\alpha| \leq d,|\gamma| \leq \kappa}$. Note that as $\alpha_{i} \geq \ell$, the equality $\alpha+\gamma=\mu+\ell \epsilon_{i}$ implies $\gamma \leq \mu$. The coefficient in the row indexed by the multiindex $\delta \leq \mu$ of length $|\delta| \leq \kappa$ and the column indexed by $\gamma \leq \mu$ of length $|\gamma| \leq \kappa$ is $z^{\mu+\ell \epsilon_{i}-\gamma} \partial^{|\delta|} z^{\gamma} /(\partial z)^{\delta}$. Its determinant is checked, as in [Păun-08], to be non-zero, for otherwise there would exist a non-zero polynomial of multidegree $\leq \mu$ and total degree $\leq \kappa$ with all derivatives of order $\leq \mu$ and total order $\leq \kappa$ vanishing. Let $P$ run over the set of polynomials in $z_{i}$ of degree $\leq \kappa$. Over the set $\left\{z_{i}^{\prime} \neq 0\right\}$, the determinant, computed by induction using $\left(z_{i}^{j}\right)^{(l)}=\left(j z_{i}^{j-1} z_{i}^{\prime}\right)^{(l-1)}=$ $j \sum_{a=0}^{l-1}\left(\begin{array}{c}l-1 \\ a\end{array}\right)\left(z_{i}^{j-1}\right)^{(a)} z_{i}^{(l-a)}$ and combinations of rows,

$$
\operatorname{det}\left(\begin{array}{ccccc}
1 & z_{i} & z_{i}^{2} & \cdots & z_{i}^{\kappa} \\
1^{\prime} & \left(z_{i}\right)^{\prime} & \left(z_{i}^{2}\right)^{\prime} & \cdots & \left(z_{i}^{\kappa}\right)^{\prime} \\
& & \vdots & & \\
& & \vdots & & \\
(1)^{(\kappa)} & \left(z_{i}\right)^{(\kappa)} & \left(z_{i}^{2}\right)^{(\kappa)} & \cdots & \left(z_{i}^{\kappa}\right)^{(\kappa)}
\end{array}\right)=1 ! 2 ! \cdots \kappa !\left(z_{i}^{\prime}\right)^{\kappa(\kappa+1) / 2}
$$

does not vanish. This shows that every vector in

$$
T\left(\mathcal{X}_{\kappa}^{F} / \mathcal{X}^{F}\right)_{\left(s_{\kappa}\left(b_{0}\right)\right)} \subset\left(T \mathcal{X}_{\kappa}^{F}\right)_{\left(s_{\kappa}\left(b_{0}\right)\right)}=T\left(\mathfrak{X}_{\kappa} / \mathbb{P}^{N}\right)_{\left(A, s_{\kappa}\left(b_{0}\right)\right)} \subset\left(T \mathfrak{X}_{\kappa}\right)_{\left(A, s_{\kappa}\left(b_{0}\right)\right)}
$$

is, up to "horizontal vectors", the value of a meromorphic vector field on $\Pi_{\kappa, 0}^{-1}\left(U^{F}\right) \subset \mathfrak{X}_{\kappa}$ holomorphic when viewed with values in $\Pi_{\kappa, 0}^{\star} \mathcal{O}_{\mathbb{P}^{n+1}}(\kappa)$.

For "horizontal vectors" (i.e. when $P \stackrel{\kappa, 0}{=} 0$ ), we use the set (6.1). By the Cramer formulae, over the set $\left\{z_{i}^{\prime} \neq 0\right\}$, for any given set of $\left(A_{\alpha}^{*}\right)_{|\alpha| \leq \kappa, \alpha \neq l \epsilon_{i}}$ there exist $\left(A_{l \epsilon_{i}}\right)_{l}$ that satisfy the previous equations. Their pole order is $\leq \kappa^{2}+2 \kappa$. The missing directions $\left(A_{\alpha}^{*}\right)_{|\alpha|>\kappa}$ are obtained with even smaller pole order, by considering some universal relations in the differential algebra of polynomials. Details for this last paragraph can be found in [Mer-09].

\section{Appendix: Using Morse inequalities for families of surfaces}

We check that in the case of surfaces, the bound $\kappa=n+1$ is optimal to find differential equations using holomorphic Morse inequalities. 
We remark first that the numbers $\mathbb{L}$ that appeared in the recursion formula for the Segre classes of the bundles $\mathcal{F}_{k}$ can be easily computed writing the Pascal triangle.

\begin{tabular}{|c|c|c|c|c|c|c|c|c|c|c|}
\hline $\mathbb{L}_{e}^{f}$ & $e=0$ & $e=1$ & $e=2$ & $e=3$ & $e=4$ & $e=5$ & $e=6$ & $e=7$ & $e=8$ & $e=9$ \\
\hline$f=0$ & 1 & & & & & & & & & \\
\hline$f=1$ & 0 & 1 & & & & & & & & \\
\hline$f=2$ & 1 & -1 & 1 & & & & & & & \\
\hline$f=3$ & 0 & 2 & -2 & 1 & & & & & & \\
\hline$f=4$ & 1 & -2 & 4 & -3 & 1 & & & & & \\
\hline$f=5$ & 0 & 3 & -6 & 7 & -4 & 1 & & & & \\
\hline$f=6$ & 1 & -3 & 9 & -13 & 11 & -5 & 1 & & & \\
\hline$f=7$ & 0 & 4 & -12 & 22 & -24 & 16 & -6 & 1 & & \\
\hline$f=8$ & 1 & -4 & 16 & -34 & 46 & -40 & 22 & -7 & 1 & \\
\hline$f=9$ & 0 & 5 & -20 & 50 & -80 & 86 & -62 & 29 & -8 & 1 \\
\hline
\end{tabular}

They also satisfy the relations

$$
\mathbb{L}_{e}^{f}-\mathbb{L}_{e+1}^{f}=\mathbb{L}_{e+1}^{f+1}
$$

7.1. On $\mathcal{X}_{1}$

We choose $\varepsilon$ to be equal to the bound we found when computing the generic nef cone of $\mathcal{X}$, that is, $\varepsilon:=r /(3 d)$. Then, we take $A=\mathcal{O}_{\mathcal{X}_{1}}(0,2 ; 1) \otimes \mathcal{O}_{\mathcal{X}}(-\varepsilon x, x)$ and $B=$ $\mathcal{O}_{\mathcal{X}}(0,2+x)$ with first Chern classes $a=\alpha_{1}+2 \alpha+x(\alpha-\varepsilon \beta)=\alpha_{1}+(2+x) \alpha-x \varepsilon \beta$ and $b=(2+x) \alpha$. We find

$$
\begin{aligned}
A^{5}-5 A^{4} B & =\left(\alpha_{1}-\varepsilon x \beta\right)^{5}-10\left(\alpha_{1}-\varepsilon x \beta\right)^{3}(2+x)^{2} \alpha^{2}-20\left(\alpha_{1}-\varepsilon x \beta\right)^{2}(2+x)^{3} \alpha^{3} \\
& =s_{3}-5 \varepsilon x s_{2} \beta-10(2+x)^{2} s_{1} \alpha^{2}-20(2+x)^{3} \alpha^{3}+30 \varepsilon(2+x)^{2} x \alpha^{2} \beta
\end{aligned}
$$

whose dominant term

$$
[-4 \chi+20 \varepsilon x] d^{2}+20\left[1-(2+x)^{2}\right] r d=-4 \chi d^{2}-20\left[3+(11 / 3) x+x^{2}\right] r d
$$

is negative.

7.2. $O n \mathcal{X}_{2}$

Here we take $A=\mathcal{O}_{\mathcal{X}_{2}}(0,6 ; 2,1) \otimes \mathcal{O}_{\mathcal{X}_{1}}(0,2 y ; y) \otimes \mathcal{O}_{\mathcal{X}}(-\varepsilon x, x)$ and $B=\mathcal{O}_{\mathcal{X}}(0,6+$ $2 y+x)$ with first Chern classes

$$
\begin{aligned}
a & =\left(\alpha_{2}+2 \alpha_{1}+6 \alpha\right)+y\left(\alpha_{1}+2 \alpha\right)+x(\alpha-\varepsilon \beta) \\
& =\alpha_{2}+(2+y) \alpha_{1}+(6+2 y+x) \alpha-\varepsilon x \beta
\end{aligned}
$$

and $b=(6+2 y+x) \alpha$. The bundle $A-B$ is $\mathcal{O}_{\mathcal{X}_{2}}(-\varepsilon x, 0 ; 2+y, 1)$.

We compute only the term $\left(A^{7}-7 A^{6} B\right)_{\text {dom }}$ in $A^{7}-7 A^{6} B$ of degree 3 in $(r, d)$. From the computation of the direct images on $\mathcal{X}$ of the Segre classes of $\mathcal{F}_{1}$, and from the Segre numbers of $\mathcal{F}_{0}$ on $\mathcal{X}$, we infer that the contributions have to contain a part in $\operatorname{seg}_{1} \operatorname{seg}_{2}$ or 
$\operatorname{seg}_{1}^{2}$ and should therefore contain only one power of $\alpha$ or $\beta$. We find that the dominant term is, viewed in $\mathcal{X}_{2}$,

$$
\begin{aligned}
\left(A^{7}-7 A^{6} B\right)_{\mathrm{dom}}= & {\left[\alpha_{2}+(2+y) \alpha_{1}\right]^{7}+7\left[\alpha_{2}+(2+y) \alpha_{1}\right]^{6}[(6+2 y+x) \alpha-\varepsilon x \beta] } \\
& -7\left[\alpha_{2}+(2+y) \alpha_{1}\right]^{6}(6+2 y+x) \alpha \\
= & {\left[\alpha_{2}+(2+y) \alpha_{1}\right]^{7}-7 \varepsilon x\left[\alpha_{2}+(2+y) \alpha_{1}\right]^{6} \beta, }
\end{aligned}
$$

and viewed in $\mathcal{X}_{1}$,

$$
\begin{aligned}
\left(A^{7}-7 A^{6} B\right)_{\operatorname{dom}}= & \operatorname{seg}_{5}\left(\mathcal{F}_{1}\right)+7(2+y) \alpha_{1} \operatorname{seg}_{4}\left(\mathcal{F}_{1}\right)-7 \varepsilon x \operatorname{seg}_{4}\left(\mathcal{F}_{1}\right) \beta \\
& +21(2+y)^{2} \alpha_{1}^{2} \operatorname{seg}_{3}\left(\mathcal{F}_{1}\right)-7 \cdot 6 \varepsilon x(2+y) \operatorname{seg}_{3}\left(\mathcal{F}_{1}\right) \alpha_{1} \beta \\
& +35(2+y)^{3} \alpha_{1}^{3} \operatorname{seg}_{2}\left(\mathcal{F}_{1}\right)-7 \cdot 15 \varepsilon x(2+y)^{2} \operatorname{seg}_{2}\left(\mathcal{F}_{1}\right) \alpha_{1}^{2} \beta \\
& +35(2+y)^{4} \alpha_{1}^{4} \operatorname{seg}_{1}\left(\mathcal{F}_{1}\right)-7 \cdot 20 \varepsilon x(2+y)^{3} \operatorname{seg}_{1}\left(\mathcal{F}_{1}\right) \alpha_{1}^{3} \beta \\
& +21(2+y)^{5} \alpha_{1}^{5}-7 \cdot 15 \varepsilon x(2+y)^{4} \alpha_{1}^{4} \beta .
\end{aligned}
$$

This leads to the following expression for the dominant term, viewed in $\mathcal{X}$ :

$$
\begin{aligned}
&\left(A^{7}-7 A^{6} B\right)_{\mathrm{dom}} \\
&= {\left[-2-14(2+y)+63(2+y)^{2}-70(2+y)^{3}+35(2+y)^{4}\right] s_{1} s_{2} } \\
&-7 \varepsilon x\left[-13+42(2+y)-45(2+y)^{2}+20(2+y)^{3}\right] s_{1}^{2} \beta \\
&= {\left[-2-14(2+y)+63(2+y)^{2}-70(2+y)^{3}+35(2+y)^{4}\right]\left(\chi d^{3}-12 r d^{2}\right) } \\
&-7 \varepsilon x\left[-13+42(2+y)-45(2+y)^{2}+20(2+y)^{3}\right] d^{3} \\
&=\left(222+518 y+483 y^{2}+210 y^{3}+35 y^{4}\right)\left(\chi d^{3}-12 r d^{2}\right) \\
&-7 \varepsilon x\left(51+102 y+75 y^{2}+20 y^{3}\right) d^{3} .
\end{aligned}
$$

We can apply the vanishing criterion provided $\varepsilon x>\chi(3+2 y)$. This leads to

$$
\begin{aligned}
\left(A^{7}-7 A^{6} B\right)_{\mathrm{dom}} \leq & \left(222+518 y+483 y^{2}+210 y^{3}+35 y^{4}\right)\left(\chi d^{3}-12 r d^{2}\right) \\
& -7 \chi(3+2 y)\left(51+102 y+75 y^{2}+20 y^{3}\right) d^{3} \\
\leq & -\left(849+2338 y+2520 y^{2}+1260 y^{3}+245 y^{4}\right) \chi d^{3} \\
& -\left(2664+6216 y+5796 y^{2}+2520 y^{3}+420 y^{4}\right) r d^{2} .
\end{aligned}
$$

\subsection{On $\mathcal{X}_{3}$}

Here we take $A$ and $B$ with first Chern classes

$$
\begin{aligned}
a= & \left(\alpha_{3}+2 \alpha_{2}+6 \alpha_{1}+18 \alpha\right)+z\left(\alpha_{2}+2 \alpha_{1}+6 \alpha\right) \\
& +y\left(\alpha_{1}+2 \alpha\right)+x(\alpha-\varepsilon \beta) \\
= & \alpha_{3}+(2+z) \alpha_{2}+(6+2 z+y) \alpha_{1}+(18+6 z+2 y+x) \alpha-\varepsilon x \beta
\end{aligned}
$$

and

$$
b=(18+6 z+2 y+x) \alpha .
$$


The bundle $A-B$ is $\mathcal{O}_{\mathcal{X}_{2}}(-\varepsilon x, 0 ; 6+2 z+y, 2+z, 1)$. In order to apply the vanishing criterion, we choose $\varepsilon x=9+3 z+y$. The dominant term of $A^{9}-9 A^{8} B$ is (computed with Maple)

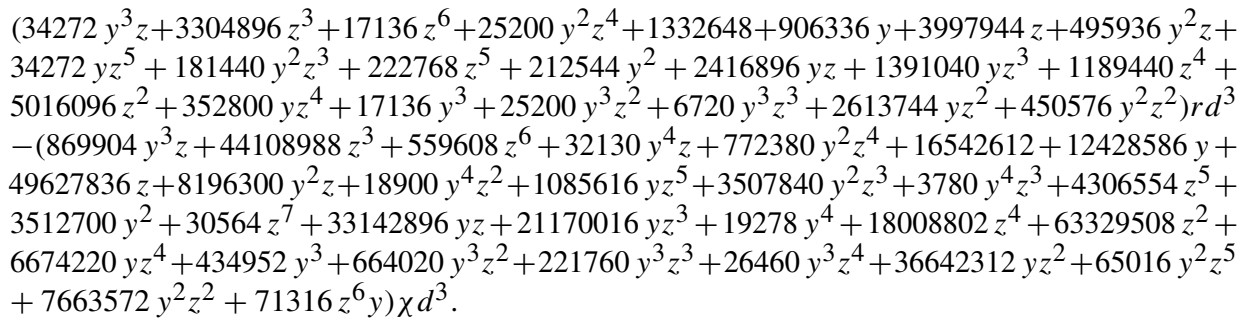

Acknowledgments. I thank Claire Voisin for the nice idea she gave me for computing nef cones. I discussed the subject of this paper with many people over more than four years. I would like to thank them all, in a single sentence.

\section{References}

[A-S-S-97] Arrondo, E., Sols, I., Speiser, R.: Global moduli for contacts. Ark. Mat. 35, 1-57 (1997) Zbl 0930.14035 MR 1443035

[Cle-86] Clemens, H.: Curves on generic hypersurfaces. Ann. Sci. École Norm. Sup. (4) 19, 629-636 (1986) Zbl 0611.14024 MR 0875091

[Dem-85] Demailly, J.-P.: Champs magnétiques et inégalités de Morse pour la $d^{\prime \prime}$-cohomologie. Ann. Inst. Fourier (Grenoble) 35, 189-229 (1985) Zbl 0565.58017 MR 0812325

[Dem-95] Demailly, J.-P.: Algebraic criteria for Kobayashi hyperbolic projective varieties and jet differentials. In: Algebraic Geometry-Santa Cruz 1995, Proc. Sympos. Pure Math. 62, Amer. Math. Soc., Providence, RI, 285-360 (1997) Zbl 0919.32014 MR 1492539

[Div-09] Diverio, S.: Existence of global invariant jet differentials on projective hypersurfaces of high degree. Math. Ann. 344, 293-315 (2009) Zbl 1166.32013 MR 2495771

[D-M-R-08] Diverio, S., Merker, J., Rousseau, E.: Effective algebraic degeneracy. Invent. Math. 180, 161-223 (2010) Zbl 1192.32014 MR 2593279

[Gra-65] Grauert, H.: Mordells Vermutung über rationale Punkte auf algebraischen Kurven und Funktionenkörper. Inst. Hautes Études Sci. Publ. Math. 25, 131-149 (1965) Zbl 0137.40503 MR 0222087

[Jou-78] Jouanolou, J.-P.: Hypersurfaces solutions d'une équation de Pfaff analytique. Math. Ann. 232, 239-245 (1978) Zbl 0354.34007 MR 0481129

[Lang-86] Lang, S.: Hyperbolic and Diophantine analysis. Bull. Amer. Math. Soc. (N.S.) 14, 159-205 (1986) Zbl 0602.14019 MR 0828820

[Man-63] Manin, Yu. I., Rational points on algebraic curves over function fields. Izv. Akad. Nauk SSSR Ser. Mat. 27, 1395-1440 (1963) (in Russian) Zbl 0178.55102 MR 0157971

[Mer-09] Merker, J.: Low pole order frames on vertical jets of the universal hypersurface. Ann. Inst. Fourier (Grenoble) 59, 1077-1104 (2009) Zbl 1172.32005 MR 2543663

[Mor-94] Moriwaki, A.: Remarks on S. Lang's conjecture over function fields. arXiv:alggeom/9412021v1 
[Mor-95] Moriwaki, A.: Geometric height inequality on varieties with ample cotangent bundles. J. Algebraic Geom. 4, 385-396 (1995) Zbl 0873.14029 MR 1311357

[Nog-81] Noguchi, J.: A higher-dimensional analogue of Mordell's conjecture over function fields. Math. Ann. 258, 207-212 (1981/82) Zbl 0459.14002 MR 0641826

[Nog-85] Noguchi, J.: Hyperbolic fibre spaces and Mordell's conjecture over function fields. Publ. Res. Inst. Math. Sci. 21, 27-46 (1985) Zbl 0583.32061 MR 0780890

[Păun-08] Păun, M.: Vector fields on the total space of hypersurfaces in the projective space and hyperbolicity. Math. Ann. 340, 875-892 (2008) Z Zl 1137.32010 MR 2372741

[Rou-07] Rousseau, E.: Weak analytic hyperbolicity of generic hypersurfaces of high degree in $\mathbb{P}^{4}$. Ann. Fac. Sci. Toulouse Math. (6) 16, 369-383 (2007) Zbl 1132.32010 MR 2331545

[Siu-04] Siu, Y.-T.: Hyperbolicity in complex geometry. In: The Legacy of Niels Henrik Abel, Springer, 543-566 (2004) Zbl 1076.32011 MR 2077584

[Tra-95] Trapani, S.: Numerical criteria for the positivity of the difference of ample divisors. Math. Z. 219, 387-401 (1995) Zbl 0828.14002 MR 1339712

[Voi-96] Voisin, C.: On a conjecture of Clemens on rational curves on hypersurfaces. J. Differential Geom. 44, 200-213 (1996) Zbl 0883.14022 MR 1669712

[Voj-78] Vojta, P.: On algebraic points on curves. Compos. Math. 78, 29-36 (1991) Zbl 0731.14015 MR 1096125 